\title{
Effect of an introductory quantum physics course using experiments with heralded photons on preuniversity students' conceptions about quantum physics
}

\author{
Philipp Bitzenbauer®* \\ Physics Education, Department of Physics, Friedrich-Alexander-Universität Erlangen-Nürnberg, \\ Staudtstr. 7, 91058 Erlangen, Germany
}

(Received 5 March 2021; accepted 29 June 2021; published 22 July 2021)

\begin{abstract}
We conducted a quasiexperimental study in order to investigate the effect of a teaching concept on quantum physics based on coincidence and correlation experiments with heralded photons on preuniversity students' conceptions of quantum physics (experimental group, $N=150$ ). We compare the results with the traditional curriculum's effect (control group, $N=130$ ) at German secondary schools using a questionnaire to assess students' conceptions of quantum physics adapted from the literature. The results show that students introduced to quantum physics using the quantum optics concept acquire conceptions of quantum physics that are significantly less influenced by classical mechanistic and deterministic conceptions than those of the control group. In more detail, correlation and principal component analysis results indicate that the conceptions acquired by experimental group students are more consistent than those of the control group students.
\end{abstract}

DOI: 10.1103/PhysRevPhysEducRes.17.020103

\section{INTRODUCTION}

For more than twenty years, teaching concepts for quantum physics have been developed in physics education research for schools and universities in order to promote a detailed conceptual understanding of quantum physics among learners. Against the backdrop of increasing technical possibilities in the preparation and detection of singlephoton states, various research groups have developed experiment-based approaches for teaching quantum physics [1-13]. At the heart of most of these experiment-based teaching sequences there are experiments on the quantum behavior of single photons at the beam splitter, so as to make nonclassical effects, e.g., photon antibunching [14] graspable. The quantum behavior of light is revealed by measuring intensity correlations of light at the outputs of a beam splitter. Such measurements were first performed by Hanbury Brown-Twiss [15]. Subsequently, they led to the development of quantum optics $[16,17]$ and were used in the laboratory by Grangier et al. [18] to unequivocally demonstrate the quantum nature of light for the first time. Marshman and Singh argue that quantum optics experiments "elegantly illustrate the fundamental concepts of quantum mechanics such as the wave-particle duality of a

*philipp.bitzenbauer@fau.de

Published by the American Physical Society under the terms of the Creative Commons Attribution 4.0 International license. Further distribution of this work must maintain attribution to the author(s) and the published article's title, journal citation, and DOI. single photon, single-photon interference, and the probabilistic nature of quantum measurement" [19] (p. 1) According to Pearson and Jackson, single-photon experiments "provide the simplest method to date for demonstrating the essential mystery of quantum physics" [10] (p. 1).

Despite such claims, few empirical studies have been published on these experiment-based teaching sequences' actual learning effectiveness. Several studies have investigated typical learning difficulties and learners' understanding of quantum physics in schools and universities [20-25], in particular, concerning quantum measurements and time evolution [26], the quantum mechanical formalism in general [27], and wave functions in one spatial dimension [28]. Nevertheless, there are still only a few studies with relatively small samples concerning learning and teaching quantum mechanics in the context of single-photon experiments [19,29,30]. Thus, although some researchers in the field have already suggested this approach, it has so far lacked consistent implementation and empirical evaluation on a large scale.

With this article, we want to contribute to closing this gap. We report on the results of a quasiexperimental comparative study. We investigate the effect of a teaching concept on quantum physics based on coincidence and correlation experiments with heralded photons on preuniversity students' conceptions of quantum physics (experimental group). Then, we compare the results with the traditional curriculum's effect (control group) at German secondary schools. In the next section, we outline the main features of the teaching concept on quantum optics, as well as the traditional curriculum (cf. Sec. II). 
We then present our research question in Sec. III, the design of our study in Sec. IV, and report on our findings in Sec. V.

\section{QUANTUM PHYSICS IN SECONDARY SCHOOL TEACHING}

\section{A. The traditional quantum physics curriculum in German secondary schools}

From their review of 15 countries' secondary school curricula on quantum physics, Stadermann et al. [31] derived an international core curriculum combining quantum physics topics that are anchored in at least 8 of the 15 nations' curricula (cf. Fig. 1). Stadermann et al. [31] conclude that at secondary schools, quantum physics is often introduced with historical experiments, and they criticize this approach: They call it quasihistorical and maintain that textbooks often only present the actual historical course of knowledge acquisition in a highly simplified way [31].

The traditional quantum physics curriculum in German secondary schools is guided by the historical development of quantum physics rather than by modern aspects or experiments and shows high correspondence to the international core curriculum in terms of content: The paper by Staderman et al. [31] lists the core elements of quantum physics curricula from six German federal states (cf. Table I).

\section{B. Learners' difficulties in traditional courses on quantum physics: A brief overview}

The traditional curricula guided by quantum physics' historical development do not really challenge the mechanistic conceptions that prevail in students' minds: "As many studies on mental models in quantum physics in the recent years have shown, ideas from classical physics are often carried over to quantum physics, causing conceptual barriers" [32] (p. 2). These conceptual barriers become

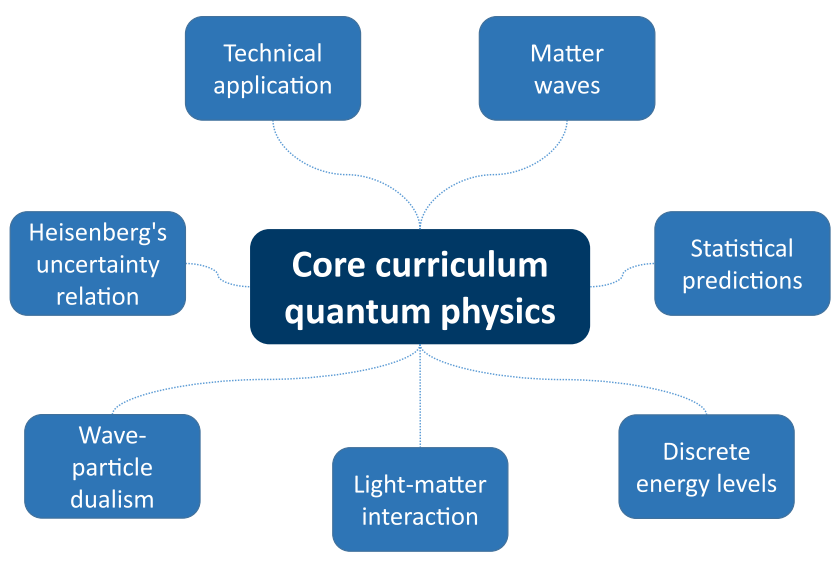

FIG. 1. Core curriculum on quantum physics for secondary school teaching as revealed by Staderman et al. [31]. particularly apparent when investigating learners' conceptions about the properties and behavior of quantum objects such as electrons or photons. For example, numerous studies have shown that learners often perceive quantum objects as classical particles with defined trajectories in the mechanical sense [22,33,34]. According to subject literature, one major reason behind this phenomenon can be found in students' understanding of models [35]. This is plausible not least because quantum physics may serve as a prime example for the need for model descriptions: Research on students' understanding of models has shown that students have difficulties distinguishing between a model and the reality described by that model [36], i.e., learners often regard "models as upscaled or downscaled exact representations of the "real thing" " [32] (p. 2). Consequently, learners often think that quantum physics models represent what quantum objects look likeconsider, for example, the common learners' conceptions of atoms among students who were exposed to Bohr's atomic model $[37,38]$. Kalkanis et al. argue that learners' misconceptions of quantum physics are caused by "the overlapping/mix-up of the conceptual frameworks of classical physics (CP) and QM" [35] (p. 257): In addition to mechanistically influenced conceptions, deterministic thinking is often the result. Such mechanistic or deterministic mental models (and hybrid conceptions) influence learners' understanding of central quantum physics concepts, e.g., the probability interpretation of quantum physics, and thus prevent a transition to quantum thinking. Quantum interference may serve as an example: Students who think of photons or electrons as small balls with a defined position moving along a well-defined path tend to believe, when conducting the double-slit experiment or an interferometer experiment with single quanta, no interference pattern will appear [23,39]. Another common students' idea is that of single quanta being reflected at the space between the two slits [40], or being deflected at a slit edge and moving away on a straight path [41].

In view of these findings, we combine two important advantages in a new introductory teaching concept on quantum physics, based on experiments with heralded photons: first, we focus on experiments that can be used to make the fundamental concepts of quantum physics accessible at secondary school level in a simple way (cf. Sec. I). Second, we aim at fostering learners' conceptions of quantum physics that are detached from mechanistic or deterministic ways of thinking.

\section{Introductory quantum physics course based on experiments with heralded photons}

Our new quantum physics curriculum for secondary schools (primary 12th grade) is based on quantum optics experiments with heralded photons. Technical details on the generation of photon pairs [42-44], on the detection and preparation of single photons via coincidence technique 
TABLE I. Quantum physics topics in the curricula of Baden-Wuerttemberg (BW), Lower Saxony (NS), North Rhine-Westphalia (NRW), Hesse (HS), Saxony (SA), and Bavaria (BY) adapted from Staderman et al. [31] without atomic physics items. Mandatory coverage of the subject in secondary school $(\checkmark)$, and optional study ( $\square$ ), respectively, are indicated. The last column allows a comparison between our new curriculum based on coincidence and correlation experiments with heralded photons, on the one hand, and the traditional curricula in terms of contents covered at German secondary schools, on the other hand. The study reported in this article was conducted at Bavarian schools. Since the different teaching approaches vary in terms of the topics covered to some extent, it is not possible to make a meaningful comparison between the approaches solely on the basis of learning gains. In this article, we will therefore refer to learners' conceptions of such quantum physics concepts that are taught in all introductory courses on quantum physics, irrespective of the content or didactic focus as discussed in Sec. III.

\begin{tabular}{lccccccc}
\hline \hline Topic & BW & NS & NRW & HS & SA & BY & New curriculum \\
\hline Light-matter interaction & $\checkmark$ & $\checkmark$ & $\checkmark$ & $\checkmark$ & $\checkmark$ & $\checkmark$ & $\checkmark$ \\
Wave-particle duality & $\checkmark$ & $\checkmark$ & $\checkmark$ & $\checkmark$ & $\checkmark$ & $\checkmark$ & $\checkmark$ \\
Matter waves & $\checkmark$ & $\checkmark$ & $\checkmark$ & $\checkmark$ & $\checkmark$ & $\checkmark$ & $\checkmark$ \\
Technical applications & & $\checkmark$ & & $\checkmark$ & $\checkmark$ & $\checkmark$ & $\checkmark$ \\
Complementarity & $\checkmark$ & $\checkmark$ & $\checkmark$ & $\checkmark$ & $\checkmark$ & $\checkmark$ & $\checkmark$ \\
Probability predictions & $\checkmark$ & $\checkmark$ & $\checkmark$ & $\checkmark$ & $\checkmark$ & $\checkmark$ & $\checkmark$ \\
Philosophical interpretations & & $\checkmark$ & $\checkmark$ & $\checkmark$ & $\checkmark$ & & $\checkmark$ \\
Tunnelling & & $\checkmark$ & & $\square$ & & $\checkmark$ & \\
Entanglement & & & & $\square$ & & $\checkmark$ & \\
Schrödinger equation & & $\checkmark$ & $\checkmark$ & & & \\
Calculation of detection probabilities & & $\checkmark$ & & & & \\
\hline \hline
\end{tabular}

$[45,46]$, and on experiments with single photons incidents on beam splitters $[8,47,48]$ have already been described in various papers, which we refer to here. Our teaching concept is divided into four lessons (of $90 \mathrm{~min}$. or eight lessons of $45 \mathrm{~min}$.), and its contents are based on the original work by Grangier et al. [18]. A detailed description of our concept and the teaching activities has already been published [29]. At this point, we only outline the key ideas of our concept. The complete teaching material can be requested from the author in both German and English.

\section{Interactive screen experiments}

We offer learners a hands-on approach to modern quantum physics experiments providing interactive screen experiments, as most schools still do not have access to quantum optics laboratories. Interactive screen experiments (ISEs) present real experiments with the help of photos from different perspectives and at different times during experimentation. They are supplemented with video or audio and experimental data from the laboratory [49]. This clearly sets them apart from mere simulations. An interactive user interface allows learners to realistically manipulate the experiment according to the principle of direct manipulation [50]. For our teaching concept, we draw on the ISEs on quantum optical real experiments by Bronner et al. [51].

Worksheets are provided for all lessons and serve to summarize the content covered. These worksheets contain problem or discussion tasks which the students solve using the ISEs. In the classroom, the ISEs can be opened either on students' PCs or on the students' own mobile devices.
For example, one of the tasks asks students to investigate the behavior of single photons at the optical beam splitter and to note observations. The students use the corresponding ISE (cf. Fig. 3) to conduct the experiment, they can view it from different perspectives, display and hide tables for documenting the measurement data, or switch devices on and off.

Within the framework of the concept under investigation, the learners work with a different interactive experiment in each of the teaching units. While the first two units are on technical details for understanding single photon experiments, lessons three and four deal with important quantum physics experiments: first, the experiment on the behavior of single photons at the beam splitter mentioned above (anticorrelation effect), followed by an experiment on the interference of single photons in the Michelson interferometer (cf. Fig. 5). Finally, the teaching series' culminates in the demonstration of the experiment by Grangier et al. [18] (cf. Fig. 2), in which the indivisibility of photons at the beam splitter is shown simultaneously with single photon interference.

\section{Key ideas of the teaching concept}

A description of the experiment of Grangier et al. [18], i.e., anticorrelation of single-photon states at the beam splitter cube or single-photon interference, is mathematically possible within the quantum mechanical formalism in order to circumvent conceptual difficulties. However, such an approach cannot be implemented in school. Therefore, we take up the thoughts from Müller and Wiesner [54], who state that for teaching quantum physics in schools, 


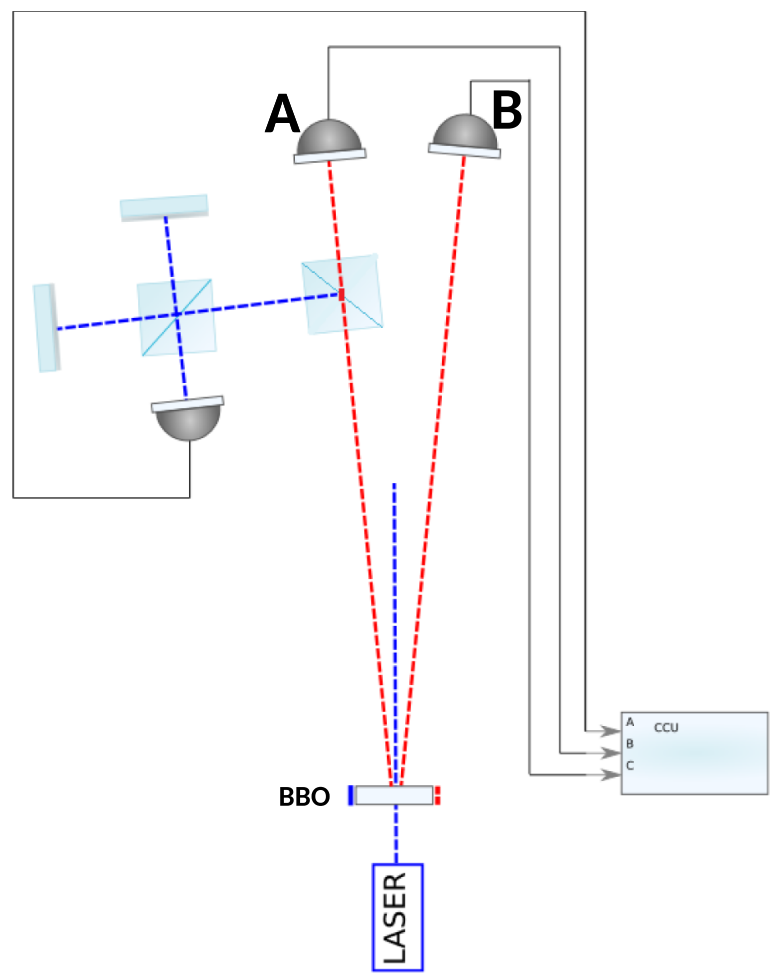

FIG. 2. Schematic setup of the experiment by Grangier et al. [18]. The experiment is based on the principle of heralded photons. If a nonlinear crystal, e.g., beta-barium borate (BBO), is irradiated with intense light from a diode laser, a quantum electrodynamic process called parametric down-conversion sets in as a reaction of this crystal to the incident light [52]. Parametric down-conversion leads to the symmetrical emission of photon pairs due to the conservation of momentum. Two detectors A and $\mathrm{B}$ are placed symmetrically facing the crystal. Simultaneous detection events, called coincident events, at the two detectors A and $\mathrm{B}$ indicate these photon pairs with high probability. A click at one detector is therefore considered a necessary prerequisite for a single photon at the second detector, or in other words: if detector $\mathrm{B}$ clicks simultaneously with $\mathrm{A}$, then $\mathrm{B}$ heralds a single photon at A. This can then be experimented with.

"conceptual clarity is even more important" [54] (p. 200). For this reason, the following three key ideas form the conceptual foundation of our teaching sequence in order to highlight differences between classical and quantum physics.

Superposition of states and statistical interpretation.Investigating true single photons at the optical beam splitter allows for simple experimental access to the concept of superposition and thus to the probabilistic nature of quantum physics [55]. In our teaching sequence, we first present the behavior of laser light at the optical beam splitter to the students in order to familiarize them with this optical component. For simplicity, we only discuss lossless beam splitters $[56,57]$ at the secondary school level. When individual photons fall on an optical beam

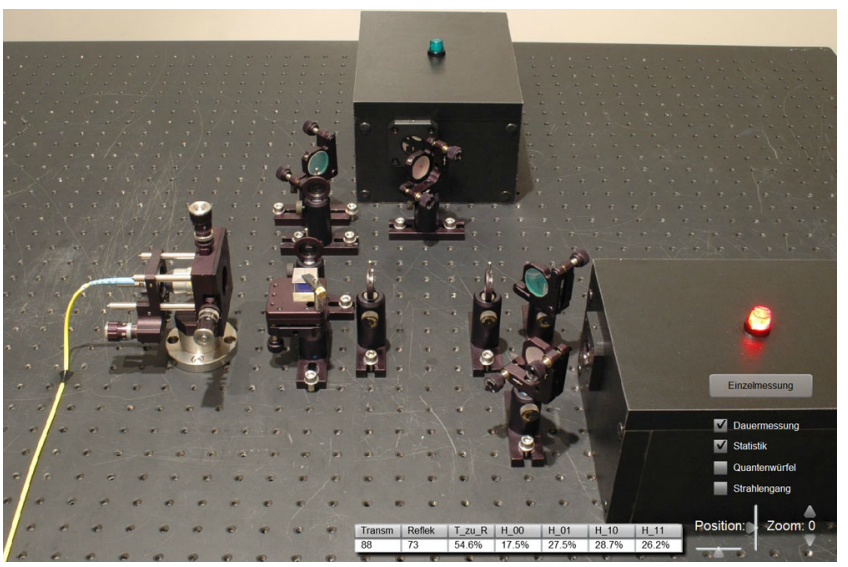

FIG. 3. Interactive screen experiment on the behavior of single photons at the beam splitter to make quantum random tangible [51].

splitter, the input state is converted into an output state that equals a superposition of transmitted and reflected. Finally, the photon is measured at precisely one of the detectors at the outputs of the optical beam splitter with equal probabilities of $50 \%$ each: "A single photon can only be detected once" [18] (p. 173). From this, our students learn that "single events are not predictable, they are random" [58] (p. 3) in quantum physics and photons are described as indivisible at the beam splitter. In an interactive screen experiment (cf. Fig. 3), students investigate the single photons' behavior at an optical beam splitter and thus understand the statistical predictability which we state as follows: "Only statistical predictions (for many repetitions) are possible in quantum physics" [58] (p. 3). In terms of quantum physics interpretations, this approach is based on quantum mechanics' ensemble interpretation [59].

The measurement process and classically well-defined dynamic properties. - Concepts of classical physics cannot simply be transferred to quantum physics-think, for example, of position: while dynamics in classical physics allow for the determination of a body's trajectory from the equations of motion, quantum objects, such as electrons or photons, do not permanently possess the property position. Thus, a quantum object has no position in the classical sense: one can speak of a quantum object's position only in the context of a measurement. This is true for all dynamical properties. Therefore, the quantum states' preparation plays a major role, and the notion of measurement occupies a central position in the concept under investigation. Teaching the quantum physical measurement process in an adequate context requires learners to understand the associated experimental components. For example, in our teaching concept, experiments are performed with binary detectors, namely, avalanche photo diodes. The detectors and all other optical components (e.g., mirrors or beam splitters) are not treated as black boxes, but are 


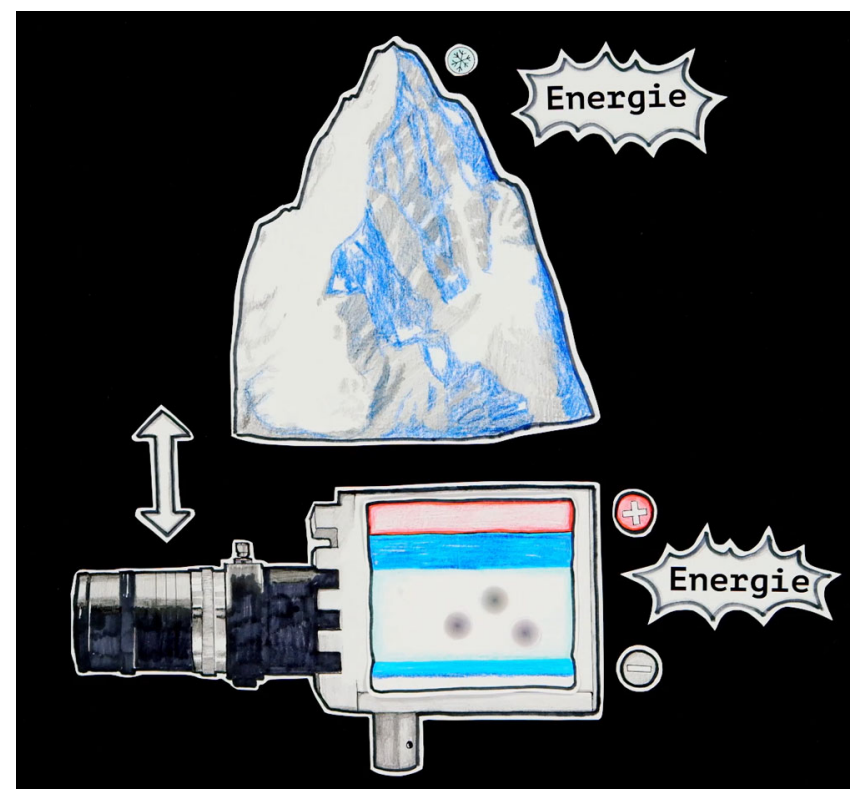

FIG. 4. Screenshot from an explanatory video: to teach students the functioning of single photon detectors at an appropriate level, we have developed an analogy to the formation and departure of snow avalanches [53]. Step by step, the video guides students through the correspondences of our analogy. In this way, technical terms such as dark count event or dead time become accessible to students. We consider the basic knowledge of such terms to be a necessary prerequisite to understanding experiments with heralded photons.

introduced at the beginning of the teaching series: For example, we establish the importance of mirrors for the adjustment of quantum optical experiments, explain to our students the basic functioning of single-photon detectors with the help of an explanatory video (cf. Fig. 4), and introduce beam splitters by means of a simple real experiment with laser light.

Quantum interference.-From the anticorrelation of single photons at the beam splitter, we conclude the indivisibility of photons. In the following, this experiment is extended by an interferometer to show single-photon interference (cf. Fig. 2). Grangier et al. [18] summarized the two experiments in their paper as follows: "Two triggered experiments have thus been performed, using the same source and the same triggering scheme for the detectors. They illustrate the wave-particle duality of light" [[18], p. 178]. The authors then continue: "Indeed, if we want to use classical concepts, or pictures, to interpret these experiments, we must use a particle picture for the first one ('the photons are not split on a beam splitter') [...]. On the contrary, we are compelled to use a wave picture ('the electromagnetic field is coherently split on a beam splitter') to interpret the second (interference) experiment" [[18], p. 178]. Our students investigate single-photon interference using an interactive screen experiment (cf. Fig. 5). Together

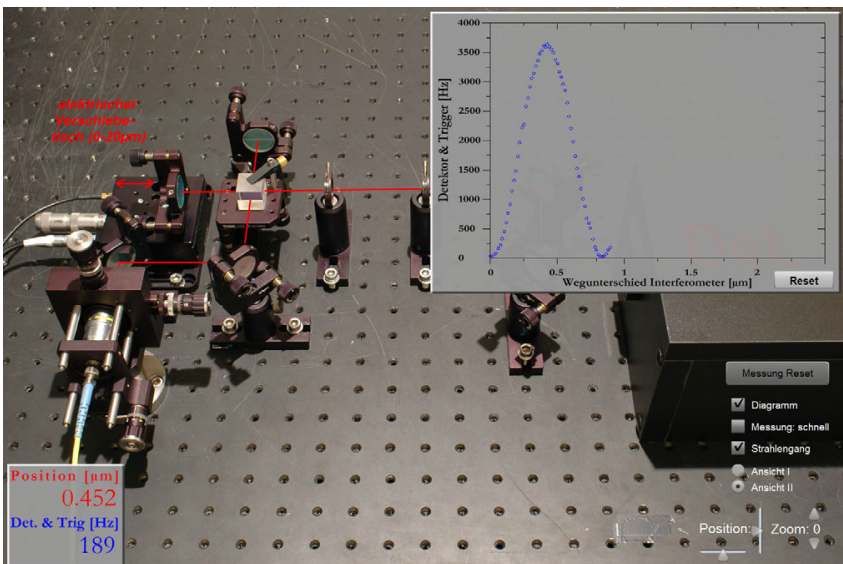

FIG. 5. Interactive screen experiment on single-photon interference [51].

with the learners, we work out that single-photon interference is a consequence of superposition and nonlocality. To emphasize a distinction from mechanistic trajectory thinking, we consider the turn of phrase as employed by Scholz et al. [60] to be appropriate: "the photon, belongs' to the whole interferometer."

Showing anticorrelation of photons and single-photon interference in one experiment towards the end of our teaching sequence, it becomes clear that a photon can neither be a particle nor a wave in the classical sense, and we discuss this with students. In summary, our approach is in accordance with a QED model of the photon: the photon is seen as an excitation of the electromagnetic field, and students develop an understanding of photons as energy quanta [61-63].

\section{RESEARCH QUESTION}

In order to compare our new quantum-optics based approach with the traditional German secondary school course on quantum physics (cf. Sec. II A.) appropriately, it is necessary to determine what construct exactly is to be assessed: In most comparative studies between traditional and new curricula (cf. $[64,65]$ ), there is agreement on what competences students should basically acquire in class, and the achievement of this goal is then compared for the different teaching proposals (control vs. treatment group). In this work, there is a particular case in this respect: Quantum optics-based approaches to quantum physics differ so fundamentally from traditional courses at schools that a comparison with other approaches is not meaningfully possible via learning gains alone. Similar arguments have also been presented in previous contributions $[40,66]$. Hence, in our study, we do not compare the effect of the two concepts on learning gains, but on learners' conceptions as developed by students introduced to quantum physics with the respective proposal (traditional vs. quantum optics). To ensure comparability, we have chosen 
two aspects of quantum physics that are taught in all introductory secondary school courses, irrespective of the content or the didactic focus: the first one is the concept of probability interpretation, and the second one is related to quantum objects' properties and behavior. Referring to the literature on students' difficulties in quantum physics (cf. Sec. II B.), we argue that these two concepts are of particular importance in promoting a classical to quantum transition in students' minds, and thus for the development of students' conceptual understanding of quantum physics.

Therefore, in this article, we investigate the extent to which secondary school students, who are introduced to quantum physics using our teaching concept on quantum optics, develop conceptions of these two quantum physics concepts (probability interpretation, quantum objects' properties and behavior) that are detached from mechanistic or deterministic thinking. We compare the results with the traditional curriculum's (cf. Sec. II A.) effect. Hence, we aim at clarifying the following research question: Are the students' conceptions

1. of quantum objects' properties and behavior less influenced by mechanistic thinking and

2. of quantum physics' probability interpretation less influenced by deterministic thinking

when students are introduced to quantum physics via coincidence and correlation experiments with heralded photons, compared to the conceptions acquired by learners in a traditional introductory quantum physics course?

In the introduction of this article, we have shown that researchers in physics education believe that quantum optics approaches are particularly suitable for making the concepts of quantum physics (QP) accessible to students. Therefore, in this article, we test the following two null hypotheses $H_{0}^{1}$ and $H_{0}^{2}$ with their respective alternative hypotheses: Students introduced to QP using the quantum optics approach based on coincidence and correlation experiments with heralded photons...

$H_{0}^{1}$ : ...do not acquire significantly less mechanistically influenced conceptions of quantum objects' properties and behavior than students introduced to $Q P$ using the traditional course.

$H_{1}^{1}$ : ...acquire significantly less mechanistically influenced conceptions of quantum objects' properties and behavior than students introduced to $Q P$ using the traditional course.

$H_{0}^{2}$ : ...do not acquire significantly less deterministically influenced conceptions of QP's probability interpretation than students introduced to $Q P$ using the traditional course.

$H_{1}^{2}$ : ...acquire significantly less deterministically influenced conceptions of $Q P$ 's probability interpretation than students introduced to $Q P$ using the traditional course.
In Sec. IV, we present our methodology in detail, explaining how we operationalized the students' conceptions of these two aspects within the context of our study, and how we test the null hypotheses presented above.

\section{METHODOLOGY}

We conducted a quasi-experimental comparative study in 12th-grade classrooms in order to contrast the learning outcomes of the traditional German teaching sequence in quantum physics (cf. Sec. II A) in a control group (short: CG) with our alternative curriculum based on a quantum optics content structure (cf. Sec. II C) in an experimental group (short: EG).

\section{A. Participants}

Our study was conducted in regular classrooms (12th grade of Bavarian secondary school) to investigate the new curriculum's effects under realistic conditions. In both, the control and the experimental group, only those students' results were included in our analysis who had filled out all the questionnaires (cf. Sec. IV B) provided during the study. Accordingly, the control group consisted of 5 classes (116 valid students out of 130 in total), and the experimental group consisted of 6 classes (139 valid students out of 150 in total). Of the 280 students surveyed in total, 126 were male, and 154 were female.

\section{B. Instruments}

\section{Assessing learning preconditions}

The scale Q2 of the German cognitive abilities test KFT 4-12 $+R$ with 20 items and $\alpha=0.83$ [67] was used to control the possible different learning preconditions between the control and the experimental group.

\section{Assessing students' conceptions of QP concepts}

For assessing students' conceptions of quantum physics, a number of instruments have been developed in physics education research [34,68-73], the majority of which, however, refer to university students as the target group. A detailed content analysis of these tests is presented in the review article by Krijtenburg-Lewerissa et al. [74]: it is shown that these concept tests contain hardly any items that are suitable for eliciting students' conceptions of quantum objects' properties and behavior or of quantum physics' probability interpretation at secondary school level. However, a broadly used instrument to assess secondary school students' conceptions of quantum physics has been developed by Mashhadi and Woolnough [75]: The authors presented an instrument consisting of "54 statements representing students' conceptions of quantum phenomena, models, and the ontological and epistemological status of theoretical entities" [[75], p. 2] as early as 1996. In this approach, students were asked to express their agreement with these statements on a five-point rating 
TABLE II. Items from the questionnaire on students' conceptions according to Müller [66] and translated into the setting single photon in the interferometer composing the scale quantum objects' properties and behavior.

\begin{tabular}{|c|c|c|}
\hline No. & Item & Weighting factor \\
\hline 1 & In an interferometer the photon behaves like a particle and like a wave. It is none of them. & $g_{1}=1$ \\
\hline 2 & $\begin{array}{l}\text { When the photon inside the interferometer moves towards the detector it takes a specific path, even if I } \\
\text { cannot determine this path. }\end{array}$ & $g_{2}=-1$ \\
\hline 3 & The photon follows a specific path, regardless of whether I observe this path or not. & $g_{3}=-1$ \\
\hline 4 & The current position of a photon between source and detector is indeterminate in principle. & $g_{4}=2$ \\
\hline 5 & $\begin{array}{l}\text { The current position of a photon between source and detector is not indeterminate in principle, but } \\
\text { unknown to the experimenter. }\end{array}$ & $g_{5}=-2$ \\
\hline 6 & $\begin{array}{l}\text { In quantum physics it is possible that a quantum object does not possess classically well-defined } \\
\text { properties, such as position. }\end{array}$ & $g_{6}=2$ \\
\hline
\end{tabular}

scale. To date, many authors have used and adapted this questionnaire by Mashhadi and Woolnough for their empirical studies on learning and teaching quantum physics: among them the frequently cited articles by Ireson $[23,76]$ or modern studies on mental models of quantum physics [32], but also studies on the evaluation of teaching concepts on quantum physics at the secondary school level $[54,66]$.

We also use items adapted from this questionnaire for our study presented here: Namely, we refer to the items in the version used by Müller and Wiesner [54] to evaluate the Munich quantum mechanics concept, because we believe that it is useful for future research on different quantum physics teaching approaches to have a large pool of results from similar studies to draw on for comparisons. While Müller and Wiesner used different item batteries to survey students' conceptions of different quantum physics topics in their evaluation study, we only use the scales on quantum objects' properties and behavior (cf. Table II) and on the probability interpretation of quantum physics (cf. Table III), as justified in Sec. III. The students involved in the study had no prior classroom experience in quantum physics. However, we still checked for possible students' conceptions prior to classroom instruction as we used our questionnaire on students' conceptions in both CG and EG, pre and post of the intervention. Thus, possible differences in learning preconditions between the control and the experimental group related to quantum physics could be excluded before the intervention (cf. Sec. VA).
The adapted questionnaire on students' conceptions that we used in our study consists of ten statements on quantum physics (sometimes also referred to as items in the following). The students were asked to rate these statements on a five-point Likert scale $(1=$ disagree, $2=$ rather disagree, $3=$ undecided, $4=$ rather agree, $5=$ agree) as suggested in the original version of the questionnaire [75]. From the students' ratings, it is possible to infer conception indices for the individual topics [66] (p. 149). These conceptual indices are standardized on a scale from -100 to +100 and thus enable an operationalization of learners' conceptions of quantum physics: An index value of +100 on a given scale means that quantum physicsadequate conceptions, detached from mechanistic (cf. statements in Table II) or deterministic (cf. statements in Table III) ways of thinking, are present. In contrast, an index value of -100 expresses that the conceptions can be regarded as mechanistic (cf. statements in Table II) or deterministic (cf. statements in Table III) [66]. A conception index of $C=0$ indicates an ambiguous attitude or a lack of knowledge to assess the items [54]. Not every item counts equally with regards to the conception value: depending on the importance, Müller [66] weighted some statements single, others double (weighting factors $g_{i} \in\{-2, \ldots, 2\} \subset \mathbb{Z}$ ). Items that are consistent with the quantum-physically adequate notion have positive weighting factors. If this is not the case, the items' weighting factors are negative [66] (p. 150). We have adopted the weighting factors set by Müller in the study presented here

TABLE III. Items from the questionnaire on students' conceptions according to Müller [66] and translated into the setting single photon in the interferometer composing the scale the probability interpretation of quantum physics.

\begin{tabular}{llc}
\hline \hline No. & Item & Weighting factor \\
\hline 1 & I cannot make statements about the behavior of single photons inside the interferometer. I can only & \\
& make statements about the statistical behavior of many identically prepared photons. & $g_{1}=2$ \\
2 & No one can tell with certainty if a photon is transmitted or reflected at a beam splitter cube. & $g_{2}=2$ \\
3 & With sufficient knowledge of the initial conditions, it would be possible to predict if a single photon is \\
& transmitted or reflected at the beam splitter. & \\
4 & With sufficient knowledge of the initial conditions, it would be possible in classical physics to predict \\
the outcome of a dice roll.
\end{tabular}


to ensure comparability. The computation of the conception index $C$ results, as explained in Ref. [66], to

$$
C=\frac{\sum_{i=1}^{n} g_{i} 50\left(v_{i}-3\right)}{\sum_{i=1}^{n}\left|g_{i}\right|},
$$

where $v_{i}$ means the respondent's rating in item $i$, i.e., $v_{i} \in\{1, \ldots, 5\}$.

The original questionnaire's items refer to the context of single electrons at the double slit [54]. We have transferred the statements to the conceptually equivalent setting of a single photon in the interferometer for our study. As a result, the content of the physical statements remains the same. Because photons and single-photon interference are covered in both the traditional course (CG) and the quantum optics course (EG), this adaptation is permissible.

The use of the questionnaire on students' conceptions in the study reported here has an essential added value: Because the individual statements neither belong to the traditional teaching concept nor to our new quantum optics course, the students' ratings are not a mere reproduction of mnemonic sentences from the intervention. Of course, students' conceptions cannot be reduced to a single index. However, the identified conception indices can be analyzed across the sample and used to assess the approach's success [66] regarding the goal of fostering students' conceptions that are detached from mechanistic or deterministic ways of thinking.

We emphasize the following: while the questionnaire we used in this study is suitable for investigating to which extent students' conceptions are influenced by mechanistic or deterministic thinking, we cannot resolve which conceptions about the queried aspects of quantum physics they develop concretely. To validate the results of this study and substantiate them qualitatively, interview studies will be conducted in the future.

\section{Design of the study}

Before starting the quantum physics lessons, the learners completed scale Q2 of the cognitive abilities test KFT 4-12 $+\mathrm{R}$ and the questionnaire on students' conceptions of quantum physics (pretest). The intervention itself took eight lessons (45 min. each) in the control group and in the experimental group, respectively. After the intervention, the students completed the questionnaire on students' conceptions (post-test).

\section{Data analysis}

Data analysis was conducted using SPSS software version 25. For descriptive statistics, we use mean values $m$ and standard deviation $\sigma$, minimum, maximum, and median. We calculate $t$ tests for independent samples to test differences between the control and the experimental group for statistical significance (cf. hypotheses in Sec. III). Each effect was considered statistically significant when the $p$ value was below the $5 \%$ threshold. We also report the effect of size measure in terms of Cohen's $d$ to judge the magnitude of statistically significant effects [77]. To visualize students' response behavior to the items in the questionnaire on students' conceptions, we resort to diverging stacked bar charts [78]. Here, a bar corresponding to $100 \%$ of student ratings is aligned relative to the scale center, with agreement corresponding to a swing of the bar to the right, and disagreement corresponding to a swing of the bar to the left. Boxplots, including median and quartiles, are used to graphically represent the distribution of students' conceptual indices for the two scales probability interpretation of quantum physics and quantum objects' properties and behavior.

For the scale on students' conceptions of quantum objects' properties and behavior (cf. Table II), we perform a principal component analysis (PCA) to uncover the underlying structure separately for the 139 students in the experimental group and for the 116 students in the control group. We retain those components resulting from PCA with eigenvalues $>1.0$ [79]. In order to identify the matrix of rotation [80], we use varimax rotation. Cronbach's $\alpha$ [81] is reported as a measure of factor reliability, and in addition we examine convergent and divergent validity. The former by calculating the average variance extracted (AVE) for each factor, the latter using the Fornell-Larcker criterion [[82], p. 46].

A PCA of the quantum physics' probability interpretation scale consisting of only four items (cf. Table III) supported the unidimensionality of this scale. Therefore, to assess the extent to which coherent visions on quantum physics' probability interpretation emerged among learners in both the control and experimental group, we report Spearman's correlation coefficient $\rho$ between the post-test ratings on different items given by the same student as intended for ordinally scaled data. For the interpretation of the correlations, we refer to [83]: weak correlation $\rho<0.20$, medium correlation $0.20 \leq \rho \leq 0.30$, strong correlation $\rho>0.30$.

Lastly, we provide a more detailed insight into the composition of the control and the experimental group. Depending on the conception index on the respective scale (quantum objects' properties and behavior and quantum physics' probability interpretation), we assign our participants from both control and experimental groups to one of three groups:

- High conception index group: Students who reach a conception index of $33.33<C \leq 100$ on a given scale are assigned this group concerning this scale. The conceptions of the students in this group seem to be predominantly detached from mechanistic (for the quantum objects' properties and behavior scale) or deterministic (for the quantum physics' probability interpretation scale) thinking.

- Medium conception index group: Students who reach a conception index of $-33.33 \leq C \leq 33.33$ on a given 
scale are assigned to this group concerning this scale. The conceptions of this groups' students are ambiguous, meaning that at least in part mechanistically (for the quantum objects' properties and behavior scale) or deterministically (for the quantum physics' probability interpretation scale) influenced thinking can be assumed.

- Low conception index group: Students who reach a conception index of $-100 \leq C<-33.33$ on a given scale are assigned to this group concerning this scale. The conceptions of this groups' students seem to be predominantly shaped by mechanistic (for the quantum objects' properties and behavior scale) or deterministic (for the quantum physics' probability interpretation scale) ways of thinking.

We assign each participant from both the control group and the experimental group to one of the three groups according to their pretest result, separately for the two scales quantum objects' properties and behavior and quantum physics' probability interpretation. In this way, we can describe exactly how the groups change towards the post-test point in time, as we repeat the group assignments based on the post-test results.

\section{RESULTS}

\section{A. Learning preconditions}

The students' percentage scores in the cognitive abilities test before the implementation of our intervention in the control group ( $m=0.62, \sigma=0.27$ ) were not statistically significantly different from those in the experimental group $(m=0.65, \sigma=0.23)$, as can be seen from a $t$ test for independent samples [ $t(253)=-1.02, p=0.309]$. Neither group had received any instruction in quantum physics before the intervention. In the questionnaire on students' conceptions of quantum physics prior to the intervention, we therefore obtained, as expected (cf. Sec. IV B.), conception indices (scale $-100, \ldots,+100$ ) close to 0 . Relative to the quantum objects' properties and behavior, the conception indices are $m=5.36, \sigma=26.06$ in the control group and $m=6.08, \sigma=27.17$ in the experimental group. This difference is not statistically significant according to a $t$ test for independent samples $[t(253)=-0.212, p=0.832]$. Related to the probability interpretation of quantum physics, the conception indices are $m=5.30, \sigma=34.06$ in the control group and $m=4.32, \sigma=37.38$ in the experimental group. Again, there is no statistically significant difference between the groups $[t(253)=0.217, p=0.829]$.

\section{B. Quantum objects' properties and behavior \\ 1. Descriptive statistics}

Table IV provides an overview of the descriptive statistics on the conception indices for the quantum objects' properties and behavior scale for control and experimental groups at both survey time points (pre and post).
TABLE IV. Descriptive statistics on the conception indices for the quantum objects' properties and behavior in control and experimental groups at both survey time points (pre and post). The number of students who achieved the respective minimum and maximum scores for both $\mathrm{CG}$ and $\mathrm{EG}$ are noted in parentheses.

\begin{tabular}{lccc}
\hline \hline & Group & CG $(N=116)$ & EG $(N=139)$ \\
\hline$m$ & pre & 5.36 & 6.08 \\
& post & 32.71 & 42.65 \\
$\sigma$ & pre & 26.06 & 27.17 \\
\multirow{4}{*}{ median } & post & 34.87 & 38.36 \\
& pre & 11.11 & 11.11 \\
$\min$ & post & 22.22 & 44.44 \\
& pre & $-72.22(3 \mathrm{x})$ & $-72.22(6 \mathrm{x})$ \\
$\max$ & post & $-50.00(1 \mathrm{x})$ & $-72.22(3 \mathrm{x})$ \\
& pre & $61.11(1 \mathrm{x})$ & $61.11(1 \mathrm{x})$ \\
& post & $100.00(3 \mathrm{x})$ & $100.00(7 \mathrm{x})$ \\
\hline \hline
\end{tabular}

\section{Comparison of control and experimental group}

From pre- to post-test we find a statistically significant increase in the students' conception indices for the scale quantum objects' properties and behavior in both the control group $[t(115)=-7.00, p<0.001 ; d=0.65]$ and the experimental group $[t(138)=-11.72, p<0.001 ; d=0.99]$ from $t$ tests for dependent samples (cf. Fig. 6). While the pretest difference between the groups has not been statistically significant (cf. Sec. VA.), we find a statistically significant difference between the control group $(m=32.71$, $\sigma=34.87)$ and the experimental group $(m=42.65, \sigma=$ $38.36)$ in the post-test $[t(253)=-2.146, \quad p=0.033$; $d=0.27]$.

Figure 7 shows the post-test distribution of the index values for the scale quantum objects' properties and behavior.

As can be seen, a large proportion of the respondents achieved an index value greater than 0 , indicating a predominant rejection of mechanistic ways of thinking at the post-test point in both groups (for the total sample: $m=38.13, \sigma=37.07)$ after the intervention. To gain more

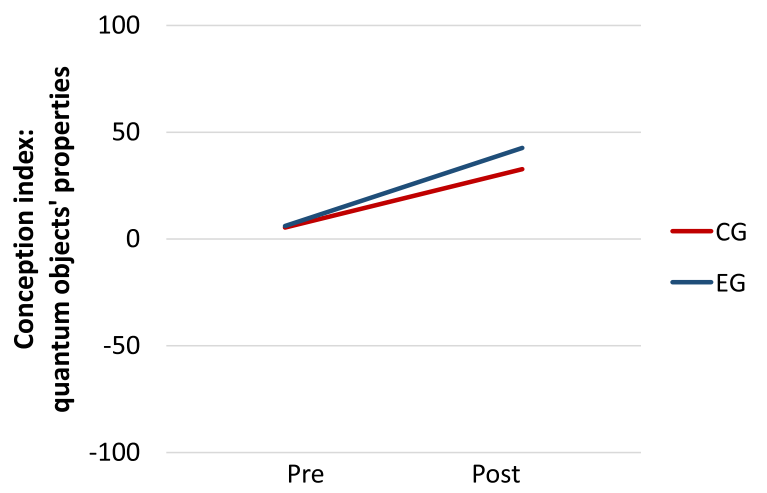

FIG. 6. We find a stronger increase of the mean conception index for quantum objects' properties and behavior from pre- to postsurvey in the experimental group than in the control group. 


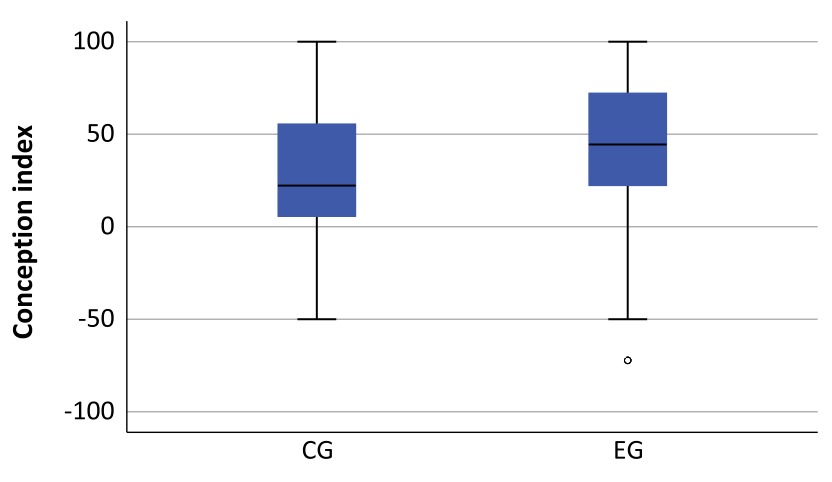

FIG. 7. Distribution of the post-test values of the conception index for the scale quantum objects' properties and behavior in CG and EG (cf. Table II).

detailed insights into the group differences we provide the distributions of the students' ratings for the individual statements: These are presented in Table V, while in Fig. 12 (cf. Appendix A), differences between control and experimental groups concerning individual statements can also be analyzed graphically.

We now describe the post-test results on a more qualitative level: Students in both groups develop an understanding that quantum objects do not permanently possess properties that can always be assigned to classical objects. For example, if we consider the response behavior to item

6. In quantum physics, it is possible that a quantum object does not possess classically well-defined properties, such as position (cf. Table II)

TABLE V. Proportion of students' agreements $(+$, rating: rather agree, agree), disagreements ( - , rating: rather disagree, disagree), and undecided votes ( $\circ$, rating: undecided) in the items of the scale on conceptions about quantum objects' properties and behavior (cf. Table II) in \%. Deviations from $100 \%$ in the row totals are due to rounding. Mean ratings with standard deviations for items in EG and CG are reported in the continuous text. Test statistics of $t$ tests for independent samples are used to examine differences in means between control and experimental groups for each item.

\begin{tabular}{lccccc}
\hline \hline No. & Group & - & $\circ$ & + & $t$ test \\
\hline 1 & CG & 5.2 & 36.2 & 58.6 & $t(253)=-0.559, p=0.576$ \\
& EG & 9.4 & 18.7 & 72.0 & \\
2 & CG & 36.2 & 19.8 & 44.0 & $t(253)=0.121, p=0.904$ \\
& EG & 35.3 & 24.5 & 40.3 & \\
3 & CG & 47.4 & 23.3 & 29.3 & $t(253)=1.918, p=0.056$ \\
& EG & 59.0 & 15.1 & 25.9 & \\
4 & CG & 12.1 & 29.3 & 58.6 & $t(253)=2.377, p=0.018 ;$ \\
& EG & 10.8 & 12.2 & 77.0 & $d=0.30$ \\
5 & CG & 38.8 & 37.1 & 24.1 & $t(253)=1.958, p=0.050 ;$ \\
& EG & 53.2 & 25.9 & 20.9 & $d=0.25$ \\
6 & CG & 4.3 & 21.6 & 74.1 & $t(253)=-0.322, p=0.747$ \\
& EG & 8.6 & 15.1 & 76.3 & \\
\hline \hline
\end{tabular}

it is striking that about three-quarters of the students agree with this (CG: $74.1 \%$, EC: $76.3 \%$ ), but only well under $10 \%$ of the students state that it is not true (CG: $4.3 \%$, EC: $8.6 \%)$. The difference in mean rating between the control group $(m=4.18, \sigma=0.97)$ and the experimental group $(m=4.22, \sigma=1.09)$ is not statistically significant $[t(253)=-0.322, \quad p=0.747] . \quad$ Apparent differences between the control and the experimental group are revealed in those items in which particular reference is made to the property position for a quantum object: $58.6 \%$ of the students in the control group agree with the statement

4. The current position of a photon between source and detector is indeterminate in principle (cf. Table II),

and it is rejected by $12.1 \%$ of the learners in the control group. $29.3 \%$ of the learners in the control group said they were undecided. On the other hand, in the experimental group, more than three-quarters of the learners, $77.0 \%$, agree with the statement, while only $12.2 \%$ are undecided, and it is rejected by $10.8 \%$. The difference in the mean ratings between the control group ( $m=3.84, \sigma=1.16)$ and the experimental group ( $m=4.18, \sigma=1.14)$ is statistically significant $[t(253)=-2.377, p=0.018 ; d=0.30]$. In particular, in the control group, many students seem to still have ambiguous conceptions about the property concept, even after traditional quantum physics instruction. For the statement

5. The current position of a photon between source and detector is not indeterminate in principle, but unknown to the experimenter ( $c f$. Table II),

$37.1 \%$ of the students in the control group state that they were undecided. Almost a quarter $(24.1 \%)$ of them believe that this statement is true, despite quantum physics lessons-only $38.8 \%$ disagree. Here, the experimental group shows a stronger detachment from mechanistic thinking, as $53.2 \%$ of the learners disagree with this statement, $25.9 \%$ are undecided, and $20.9 \%$ agree with it. The difference in the mean ratings between the control group ( $m=2.71, \sigma=1.23$ ) and the experimental group $(m=2.39, \sigma=1.34)$ is statistically significant by trend $[t(253)=1.958, p=0.050 ; d=0.25]$.

The statement

2. When the photon inside the interferometer moves towards the detector it takes a specific path, even if I cannot determine this path (cf. Table II),

and the statement

3. The photon follows a specific path, regardless of whether I observe this path or not (cf. Table II), 
TABLE VI. Factor loadings for our two-factor PCA solution for both datasets from experimental and control group. Retained factor loadings are in boldface for each item per group.

\begin{tabular}{|c|c|c|c|c|}
\hline \multirow[b]{2}{*}{ Items by factor } & \multicolumn{2}{|c|}{ Experimental group } & \multicolumn{2}{|c|}{ Control group } \\
\hline & Factor 1 & Factor 2 & Factor 1 & Factor 2 \\
\hline \multicolumn{5}{|l|}{ Factor 1: Properties of quantum objects } \\
\hline $\begin{array}{l}\text { 1. In an interferometer the photon behaves like a particle and like a wave. It is } \\
\text { none of them. }\end{array}$ & 0.69 & 0.29 & 0.32 & 0.28 \\
\hline $\begin{array}{l}\text { 4. The current position of a photon between source and detector is indeterminate } \\
\text { in principle. }\end{array}$ & 0.73 & -0.12 & 0.79 & -0.26 \\
\hline $\begin{array}{l}\text { 5. The current position of a photon between source and detector is not } \\
\text { indeterminate in principle, but unknown to the experimenter. }\end{array}$ & 0.82 & -0.09 & 0.74 & 0.29 \\
\hline $\begin{array}{l}\text { 6. In quantum physics it is possible that a quantum object does not possess } \\
\text { classically well-defined properties, such as position. }\end{array}$ & 0.77 & 0.24 & 0.54 & 0.20 \\
\hline \multicolumn{5}{|l|}{ Factor 2: Behavior of quantum objects } \\
\hline $\begin{array}{l}\text { 2. When the photon inside the interferometer moves towards the detector it takes } \\
\text { a specific path, even if I cannot determine this path. }\end{array}$ & 0.04 & 0.74 & -0.06 & 0.88 \\
\hline $\begin{array}{l}\text { 3. The photon follows a specific path, regardless of whether I observe this path } \\
\text { or not. }\end{array}$ & 0.05 & 0.80 & 0.40 & 0.64 \\
\hline Eigenvalues & 2.34 & 1.26 & 2.05 & 1.14 \\
\hline$\%$ variance & $39.01 \%$ & $21.04 \%$ & $34.22 \%$ & $19.05 \%$ \\
\hline
\end{tabular}

both address the idea that quantum objects move along fixed mechanical paths. The students' responses to these statements are ambiguous, which makes qualitative followup studies even more important: Around 35\% of all students surveyed disagree with statement 2 (CG: $36.2 \%$, EG: $35.3 \%$ ), but even more than $40 \%$ of all students agree with this statement (CG: $44.0 \%$, EG: $40.3 \%$ ). The mean ratings between control group $(m=2.97, \sigma=1.37)$ and experimental group $(m=2.99, \sigma=1.29)$ are not statistically significantly different $[t(253)=0.121, p=0.904]$. $59.0 \%$ of the students in the experimental group disagree with statement 3-a larger proportion than students in the control group (47.4\%). The difference in mean ratings between the control group ( $m=2.70, \sigma=1.37)$ and the experimental group $(m=2.37, \sigma=1.32)$ on statement 3 is statistically significant by trend $[t(253)=1.918$, $p=0.056]$.

A principal component analysis (taking into account correlations between student answers on different items) was conducted to uncover the underlying structure of the items of the quantum objects' properties and behavior scale and thus to gain deeper insights into the consistency of student responses.

\section{Principal component analysis}

We conducted a principal component analysis with varimax rotation for both the control groups' and the experimental groups' responses on the quantum objects' properties and behavior scale. For both samples, the datasets were appropriate for PCA taking into account the Kaiser-Mayer-Olkin criterion (EG: $\mathrm{KMO}=0.701$; $\mathrm{CG}: \mathrm{KMO}=0.568)$ and Bartlett's test of sphericity $\left[\mathrm{EG}: \chi^{2}(15)=146.788, p<0.001 ; \mathrm{CG}: \chi^{2}(15)=91.807\right.$, $p<0.001]$.
The criteria eigenvalue $>1.0$, explained variance $>10 \%$ per factor, and scree plot bend led to a two-factor solution for both datasets (cf. Table VI). In terms of content these two factors are interpretable: factor 1 consists of four statements that address properties of quantum objects, while factor 2 consists of two items that address quantum objects' behavior (here: photons) from the students' point of view. This empirical factor structure is in line with the expectation. The two-factor solution accounted for $53.27 \%$ of the total variance in all items for the control group and for $60.05 \%$ of the total variance in all items for the experimental group. In Table VI, all factor loadings are shown.

The fundamental factor structure does not differ for the two samples, although it is noticeable that we find higher factor loadings for the PCA results from the experimental groups' dataset than from the control groups' dataset and vice versa for secondary loadings. This can be considered as a first indication that among the learners of the experimental group (quantum-optics based teaching approach), more consistent conceptions on quantum objects' properties and behavior emerge in comparison to the conceptions of control group students (traditional curriculum). This observation is supported by analyzing the internal consistency of the factors: whereas Cronbach's $\alpha$ as an estimator of internal consistency of factor 1 is 0.75 for the experimental groups' data, it shows to be 0.52 for the control group. For two-item scales, like factor 2 in our case, the Spearman-Brown coefficient was found to be "the most appropriate reliability coefficient" [84] (p. 641) because the "coefficient alpha almost always underestimates true reliability" [84] (p. 641). We found the Spearman-Brown coefficient to be 0.41 for the experimental group dataset and to be 0.53 for the control group 
TABLE VII. Descriptive statistics on the post-test conception indices for factor 1 properties of quantum objects and factor 2 quantum objects' behavior retained from PCA for control and experimental group students, respectively. The number of students who achieved the respective minimum and maximum scores for both CG and EG are noted in parentheses.

\begin{tabular}{lccccc}
\hline \hline & \multicolumn{2}{c}{$\mathrm{CG}(N=116)$} & & \multicolumn{2}{c}{$\mathrm{EG}(N=139)$} \\
\cline { 2 - 3 } \cline { 6 - 6 } & Factor 1 & Factor 2 & & Factor 1 & Factor 2 \\
\hline$m$ & 39.66 & 8.41 & & 50.26 & 16.01 \\
$\sigma$ & 37.16 & 56.52 & & 45.26 & 51.69 \\
median & 42.86 & 0.00 & & 50.00 & 25.00 \\
min & -42.86 & -100.00 & & -92.86 & -100.00 \\
& $(1 \mathrm{x})$ & $(4 \mathrm{x})$ & & $(1 \mathrm{x})$ & $(3 \mathrm{x})$ \\
$\max$ & 100.00 & 100.00 & & 100.00 & 100.00 \\
& $(14 \mathrm{x})$ & $(12 \mathrm{x})$ & & $(31 \mathrm{x})$ & $(15 \mathrm{x})$ \\
\hline \hline
\end{tabular}

dataset. These low values are no surprise due to the length of this scale.

We found stronger evidence for convergent validity of the two-factor model for the experimental group than for the control group: the AVE values are above the acceptable value of 0.5 for both factor $1(\mathrm{AVE}=0.56)$ and factor 2 $(\mathrm{AVE}=0.59)$ in the experimental group dataset. For the control group, the AVE value for factor 1 is $\mathrm{AVE}=0.39$ and for factor 2 it is $\mathrm{AVE}=0.59$. This can still be regarded sufficient if the reliability coefficient does not undercut 0.6 [85]. The Fornell-Larcker criterion [82] (p. 46) is met because the squared correlations of the two factors (EG: $\rho^{2}=0.02$; CG: $\left.\rho^{2}=0.11\right)$ are smaller than the AVE per factor for both datasets supporting divergent validity.

Lastly, we calculated the conception indices of both experimental and control group students for the two factors properties of quantum objects (factor 1) and quantum objects' behavior (factor 2), respectively. Descriptive statistics for both retained components are presented in Table VII. The results show quite clearly: a large proportion of the learners was able to at least predominantly overcome mechanistic ways of thinking about the quantum objects' properties (factor 1) towards the post-test point in time. However, the experimental group students $(m=50.26$, $\sigma=45.26)$ outperform control group students $(m=39.66$, $\sigma=37.16$ ): The difference in conception indices is statistically significant at the 5\% level after a $t$ test for independent samples $[t(253)=-2.018, p=0.045 ; d=0.25]$. The descriptive statistics on factor 2 , however, underline the persistence of mechanistic thinking with regard to the quantum objects' behavior: here, the conception indices on quantum objects' behavior (cf. factor 2 in Table VII) are close to 0 in both the control group ( $m=8.41, \sigma=56.52)$ and the experimental group ( $m=16.01, \sigma=51.69)$, indicating at least ambiguous conceptions among learners in both groups-even after the intervention. This observation originates in and is supported by the fact that the proportion of students surveyed who agree with statements 2 and 3 is about the same as the proportion of students surveyed who disagree with statements 2 and 3 (cf. Table V). Thus, the learners do not seem to have completely turned away from their mechanistic thinking in classical paths. In particular, the large standard deviations indicate a wide spread among the learners of both groups. This is in accordance with the results presented in the previous section. The mean conception indices in the experimental group are slightly higher than those in the control group, but this difference does not prove to be statistically significant $[t(253)=$ $-1.121, p=0.264]$. Of course, follow-up studies using more suitable instruments have to be conducted in order to support the findings presented here (cf. Sec. VII).

\section{Composition of control and experimental group}

In a final step of the analysis, we divided the participants into three groups, depending on their conception indices on the quantum objects' properties and behavior scale (cf. Table II): 1. a high conception index group including participants with conception indices $C>33.33$, 2. a medium conception index group including participants with conception indices $-33.33 \leq C \leq 33.33$, and 3 . a low conception index group including participants with conception indices $C<-33.33$. We did this separately for the preand the post-test results. We present descriptive statistics which reveal differences between the high, medium, and low conception index groups' students within the control group and the experimental group, respectively. Furthermore, this approach also reveals differences between the compositions of control and experimental group. For a more detailed analysis, we visualize transitions between the high, medium, and low conception index groups from pre- to post-test including the exact number of participants in each of the three groups for CG and EG, respectively (cf. Fig. 8).

Table VIII provides an overview of descriptive statistics for the high, medium, and low conception index groups divided by pre- and post-test for CG and EG, respectively. The mean conception indices (and the corresponding standard deviations) for the three groups $\mathrm{HC}, \mathrm{MC}$, and LC hardly differ between the control and experimental group at the post-test point in time. However, the proportion of students belonging to the high conception index group at the post-test time in point is significantly higher for the experimental group $(61.15 \%)$ than for the control group (CG: $41.38 \%$ ). Consequently, almost $60 \%$ of the control group students $(57.76 \%)$ are assigned to the medium conception index group on the basis of their post-test results, which only applies to $34.53 \%$ of the experimental group students. This observation thus indicates the following: It seems that the quantum-optics based teaching approach used to introduce the experimental group to quantum physics promotes conceptions detached from mechanistic thinking among a larger proportion of the learners than is the case using traditional quantum physics curricula oriented to quantum physics' historical 


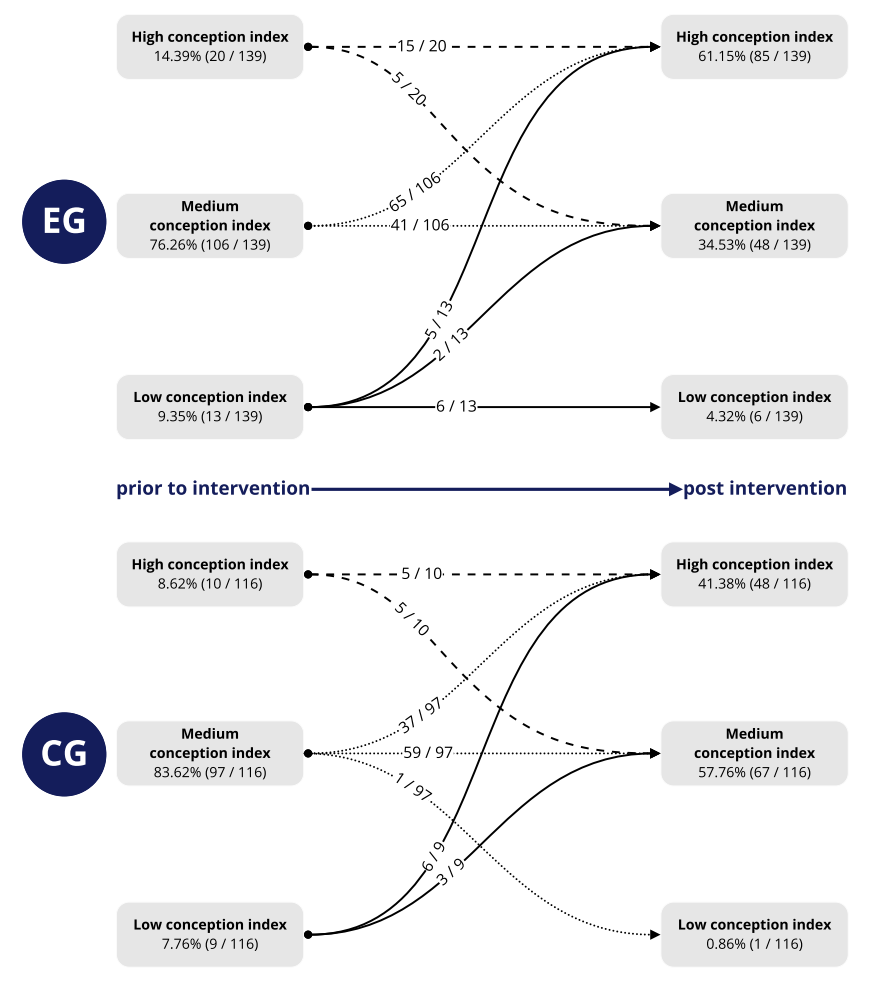

FIG. 8. Students' transitions between high (HC), medium (MC), and low (LC) conception index groups from pre- to post-test based on the students' results on the quantum objects' properties and behavior scale. It is shown which number of students from each of the pretest groups $\mathrm{HC}, \mathrm{MC}$, and LC change to which group at the post-test point in time. For both survey time points, it is also indicated how many students are assigned to the three groups in total, for both EG and CG.

development. Of course, this finding needs to be verified in follow-up studies.

However, Fig. 8 also provides graphical illustration of this observation: $40.57 \%$ of the control group students who

TABLE VIII. Share of students (\% stud.) per group (HC: high conception index group, MC: medium conception index group, LC: low conception index group) based on the pre- and post-test results on the quantum objects' properties and behavior scale for $\mathrm{CG}$ and EG, respectively. Mean values $m$ and standard deviations $\sigma$ of the conception indices are provided.

\begin{tabular}{|c|c|c|c|c|c|}
\hline & & \multicolumn{2}{|c|}{$\mathrm{CG}(N=116)$} & \multicolumn{2}{|c|}{$\mathrm{EG}(N=139)$} \\
\hline & & pre-test & post-test & pre-test & post-test \\
\hline $\mathrm{HC}$ & $\begin{array}{c}\% \text { stud. } \\
m \\
\sigma\end{array}$ & $\begin{array}{c}8.62 \% \\
46.11 \\
8.30\end{array}$ & $\begin{array}{c}41.38 \% \\
68.52 \\
18.56\end{array}$ & $\begin{array}{c}14.39 \% \\
43.33 \\
7.34\end{array}$ & $\begin{array}{c}61.15 \% \\
67.58 \\
18.76\end{array}$ \\
\hline $\mathrm{MC}$ & $\begin{array}{c}\% \text { stud. } \\
m \\
\sigma\end{array}$ & $\begin{array}{c}83.62 \% \\
6.82 \\
16.44\end{array}$ & $\begin{array}{c}57.76 \% \\
8.29 \\
15.21\end{array}$ & $\begin{array}{c}76.26 \% \\
6.66 \\
13.68\end{array}$ & $\begin{array}{c}34.53 \% \\
11.00 \\
15.31\end{array}$ \\
\hline $\mathrm{LC}$ & $\begin{array}{c}\% \text { stud. } \\
m \\
\sigma\end{array}$ & $\begin{array}{c}7.76 \% \\
-55.56 \\
12.73\end{array}$ & $\begin{array}{c}0.86 \% \\
-50.00 \\
0.00\end{array}$ & $\begin{array}{c}9.35 \% \\
-55.98 \\
16.74\end{array}$ & $\begin{array}{l}4.32 \% \\
-57.41 \\
17.09\end{array}$ \\
\hline
\end{tabular}

have been assigned to either the medium (37 out of 97) or the low conception index group (6 out of 9 ) at the pre-test point in time changed to the high conception index group based on the post-test results. In the experimental group, however, this even applies to $58.82 \%$ of the students who were assigned to either the medium (65 out of 106) or the low conception index group ( 5 out of 13 ) at the pretest point in time.

\section{Probability interpretation of quantum physics \\ 1. Descriptive statistics}

Table IX provides an overview of the descriptive statistics for the conception indices on the probability interpretation of quantum physics scale for control and experimental groups at both survey time points (pre and post).

\section{Comparison of control and experimental group}

From pre- to post-test we find a statistically significant increase in the students' conception indices for the scale probability interpretation of quantum physics in both the control group $[t(115)=-7.61, p<0.001 ; d=0.71]$ and the experimental group $[t(138)=-11.68, p<0.001 ; d=0.99]$ from $t$ tests for dependent samples (cf. Fig. 9). While the pretest difference between the groups has not been statistically significant (cf. Sec. VA.), we find a statistically significant difference between the control group ( $m=34.98, \sigma=$ $42.00)$ and the experimental group $(m=50.51, \sigma=39.24)$ in the post-test $[t(253)=-3.049, p=0.003 ; d=0.38]$.

Figure 10 shows the post-test distribution of index values for the probability interpretation of quantum physics scale.

It can be seen that a large proportion of the respondents achieved a conception index greater than 0 , indicating the presence of quantum physically adequate ideas in both groups (for the total sample: $m=43.46, \sigma=41.18$ ). More detailed insights into the group differences are provided by the distributions of the students' ratings for the individual items. These are presented in Table X, and in Fig. 13 (cf. Appendix B), differences between control and experimental groups can also be analyzed graphically.

TABLE IX. Descriptive statistics on the conception indices for the probability interpretation of quantum physics in control and experimental groups at both survey time points (pre and post).

\begin{tabular}{lccc}
\hline \hline & Group & CG & EG \\
\hline$m$ & pre & 5.30 & 4.32 \\
& post & 34.98 & 50.51 \\
$\sigma$ & pre & 34.06 & 37.38 \\
\multirow{4}{*}{ median } & post & 42.00 & 39.24 \\
& pre & 3.57 & 0.00 \\
min & post & 42.86 & 57.14 \\
& pre & $-85.71(3 \mathrm{x})$ & $-85.71(5 \mathrm{x})$ \\
$\max$ & post & $-85.71(2 \mathrm{x})$ & $-50.00(1 \mathrm{x})$ \\
& pre & $85.71(3 \mathrm{x})$ & $85.71(6 \mathrm{x})$ \\
& post & $100.00(5 \mathrm{x})$ & $100.00(24 \mathrm{x})$ \\
\hline \hline
\end{tabular}


100

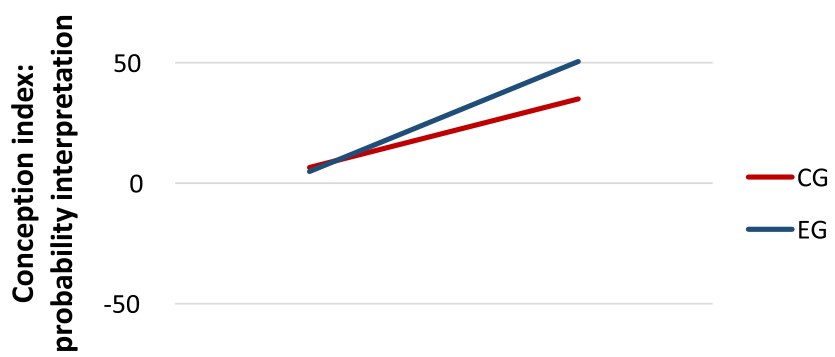

$-100$

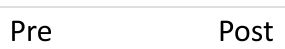

FIG. 9. We find a stronger increase of the mean conception index for the probability interpretation of quantum physics from pre- to postsurvey in the experimental group than in the control group.

We now describe the post-test results on a more qualitative level: If one looks more closely at the response behavior for individual items, it becomes apparent that there is a prevailing rejection of deterministic concepts in both groups. The majority of the respondents seems to be aware of the difference to classical physics if one considers the two statements

3. With sufficient knowledge of the initial conditions, it would be possible to predict if a single photon is transmitted or reflected at the beam splitter (cf. Table III)

and

4. With sufficient knowledge of the initial conditions, it would be possible in classical physics to predict the outcome of a dice roll (cf. Table III).

of the questionnaire on students' conceptions: Both in the control group $(60.3 \%)$ and the experimental group $(67.6 \%)$, the majority of the students reject statement 3 . Only around $15 \%$ of the learners agree with statement 3 and thus believe

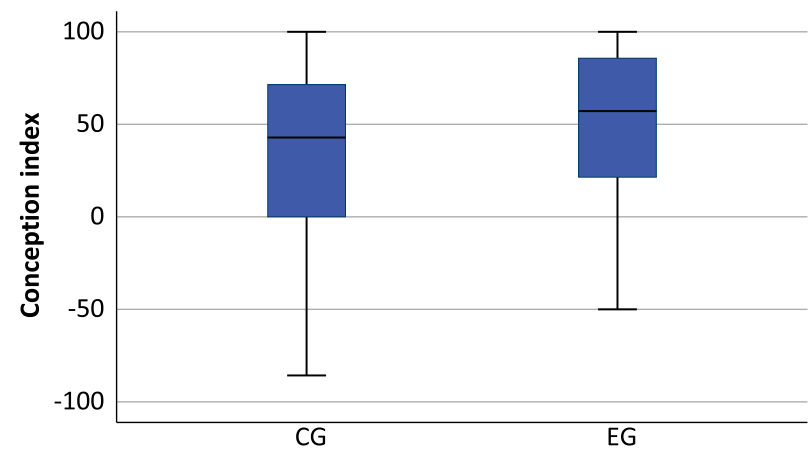

FIG. 10. Distribution of the post-test values of the conception index for the scale probability interpretation of quantum physics in CG and EG (cf. Table III).
TABLE X. Proportion of students' agreements (+, rating: rather agree, agree), disagreements ( - , rating: rather disagree, disagree), and undecided votes ( $\circ$, rating: undecided) in the items of the scale on conceptions about the probability interpretation of quantum physics (cf. Table III) in \%. Deviations from $100 \%$ in the row totals are due to rounding. Test statistics of $t$ tests for independent samples are used to examine differences in means between control and experimental groups for each item.

\begin{tabular}{lccccc}
\hline \hline No. & group & - & $\circ$ & + & $t$-test \\
\hline 1 & CG & 19.0 & 28.4 & 52.6 & $t(253)=-3.144, p=0.002$ \\
& EG & 10.1 & 23.7 & 66.2 & $d=0.39$ \\
2 & CG & 21.6 & 20.7 & 57.8 & $t(253)=-1.671, p=0.096$ \\
& EG & 13.7 & 12.2 & 74.1 & \\
3 & CG & 60.3 & 21.6 & 18.1 & $t(253)=0.980, p=0.328$ \\
& EG & 67.6 & 16.5 & 15.3 & \\
4 & CG & 13.8 & 20.7 & 65.5 & $t(253)=-2.721, p=0.007 ;$ \\
& EG & 6.5 & 14.4 & 79.2 & $d=0.34$ \\
\hline \hline
\end{tabular}

that one can predict whether a single photon will be transmitted or reflected at the beam splitter with sufficient knowledge of initial conditions (CG: 18.1\%, EG: 15.3\%). The difference in mean ratings between the control group ( $m=2.16, \sigma=1.29)$ and the experimental group ( $m=2.01$, $\sigma=1.26)$ is not statistically significant for item 3 $[t(253)=0.980, p=0.328]$. In the fourth item, the response behavior reverses, as expected. However, the proportion of learners who disagree (CG: $13.8 \%$, EG: $6.5 \%$ ) or are undecided (CG: $20.7 \%$, EG: $14.4 \%$ ) is higher in the control group than in the experimental group. Consequently, only $65.5 \%$ of the control group learners, but almost four-fifths of the experimental group learners (79.2\%) agreed with the statement. In this fourth item, there is a statistically significant difference in mean rating between the control group ( $m=3.95, \sigma=1.39)$ and the experimental group $(m=4.36, \sigma=1.02)$ according to a $t$ test $[t(253)=-2.721, p=0.007 ; d=0.34]$. To obtain an assessment of the consistency of students' responses on these two items, we calculated Spearman's correlation: Between the ratings of the control group students on these two items, we find a statistically significant correlation of $\rho=-0.47$ ( $p<0.001)$. For the ratings of the experimental group students, this is only slightly lower, at $\rho=-0.42$ $(p<0.001)$. Both correlations can be considered high according to Ref. [83]. As a result, we can assume consistent conceptions among both groups in this respect. However, the ratings of the experimental groups' students seem to be less influenced by deterministic thinking on average, as we can see from the response behavior (cf. Table $\mathrm{X}$ ) and the mean ratings.

Moreover, a large proportion of the students in both groups appear to have gained an idea of the statistical nature of quantum physics. They seem to be aware that they cannot make statements for a single quantum object, but only for an ensemble of quantum objects. This is underlined by the students' response behavior on items 1 and 2, indicating that the experimental group students' conceptions might indeed 
be less influenced by deterministic thinking than those of the control group students at the post-test point in time:

\section{I cannot make statements about the behavior of single photons inside the interferometer. I can only make statements about the statistical behavior of many sim- ilarly prepared photons ( $c f$. Table III).}

While $52.6 \%$ of the students in the control group agree with the statement (19.0\% disagree), this is even true for $66.2 \%$ of the learners in the experimental group (10.1\% disagree). The difference in mean rating between the control group $(m=3.41, \sigma=1.24)$ and the experimental group ( $m=3.84, \sigma=0.97)$ is statistically significant $[t(253)=-3.144, p=0.002 ; d=0.39]$.

For item 2, a similar response behavior is observed: $21.6 \%$ of the control group students disagree with the statement

2. No one can tell with certainty if a photon is transmitted or reflected at a beam splitter cube (cf. Table III),

but only $13.7 \%$ of the experimental group students. Conversely, only $60 \%$ of the control group agree with this statement (57.8\%), while almost three-quarters of all students in the experimental group agree with this statement (74.1\%). The difference between the mean ratings of the experimental group students ( $m=4.02, \sigma=1.29)$ and the control group students ( $m=3.73, \sigma=1.47)$ is statistically significant by trend $[t(253)=-1.671, p=0.096]$ for this item. To obtain an assessment of the consistency of students' responses on the items 1 and 2, we calculated Spearman's correlation again: we found no correlation between the ratings of the control group students on these two items $(\rho=0.09, p=0.319)$. This indicates that there is no consistency in the control group students' response behavior on these two items. Whether there is indeed little or no consistency in the control group students' conceptions of the probabilistic interpretation of quantum physics cannot be determined looking only at these two items, but it is at least (a) questionable on the basis of the results presented here and (b) in accordance with the literature on students' difficulties in traditional curricula (cf. Sec. II B.). Between the ratings on items 1 and 2 of the experimental group students, however, we find a significant correlation of $\rho=0.20(p=0.018)$. According to Ref. [83], this can be regarded as a medium correlation. As a result, we can assume consistent conceptions among the experimental groups' students in this respect. Taken together, the ratings of the experimental groups' students seem to be somewhat less influenced by deterministic thinking on average, as we can see from the response behavior (cf. Table $\mathrm{X}$ ) and the mean ratings presented in this section.

\section{Composition of control and experimental group}

In a final step of the analysis, we again divided the participants into three groups depending on their conception indices on the probability interpretation of quantum physics scale (cf. Table III): 1 . a high conception index group, 2. a medium conception index group, and 3. a low conception index group including participants. We did this separately for the pre- and the post-test results. Table XI provides an overview of descriptive statistics for the high, medium, and low conception index groups divided by pre- and post-test for CG and EG, respectively.

Unlike in the case of the scale quantum physics' properties and behavior, we see clear differences between the experimental and the control group with regard to the post-test mean values in the groups $\mathrm{HC}, \mathrm{MC}$, and LC here: For example, the conception indices' mean values at the post-test point in time differ between the experimental and control groups by almost 7 points in the HC (CG: 65.26; EG: 72.02), and by almost 8 points in the MC (CG: 2.06 ; EG: 6.07). The proportion of students belonging to the high conception index group at the post-test point in time is significantly higher for the experimental group (69.06\%) than for the control group (CG: 56.90\%). Consequently, almost $40 \%$ of the control group students $(38.79 \%)$ are assigned to the medium conception index group on the basis of their post-test results, which only applies to $28.78 \%$ of the experimental group students. Thus, the results indicate that the quantum-optics based teaching approach supports a larger proportion of students in developing conceptions of quantum physics' probability interpretation detached from deterministic thinking than is the case using traditional quantum physics curricula guided by quantum physics' historical development. Of course, this finding needs to be verified in follow-up studies.

Nevertheless, Fig. 11 also provides graphical illustration of this observation: $51.61 \%$ of the control group students who have been assigned either the medium (44 out of 81) or the low conception index group (4 out of 12) at the pre-test point in time changed to the high conception index group

TABLE XI. Share of students (\% stud.) per group (HC: high conception index group, MC: medium conception index group, LC: low conception index group) based on the pre- and post-test results on the quantum physics' probability interpretation scale for $\mathrm{CG}$ and $\mathrm{EG}$, respectively. Mean values $m$ and standard deviations $\sigma$ of the conception indices are provided.

\begin{tabular}{|c|c|c|c|c|c|}
\hline & & \multicolumn{2}{|c|}{$\mathrm{CG}(N=116)$} & \multicolumn{2}{|c|}{$\mathrm{EG}(N=139)$} \\
\hline & & pretest & post-test & pretest & post-test \\
\hline $\mathrm{HC}$ & $\begin{array}{c}\% \text { stud. } \\
m \\
\sigma\end{array}$ & $\begin{array}{c}19.83 \% \\
51.55 \\
17.09\end{array}$ & $\begin{array}{c}56.90 \% \\
65.26 \\
20.20\end{array}$ & $\begin{array}{c}24.46 \% \\
51.68 \\
17.76\end{array}$ & $\begin{array}{c}69.06 \% \\
72.02 \\
22.58\end{array}$ \\
\hline $\mathrm{MC}$ & $\begin{array}{c}\% \text { stud. } \\
m \\
\sigma\end{array}$ & $\begin{array}{c}69.83 \% \\
2.03 \\
16.22\end{array}$ & $\begin{array}{c}38.79 \% \\
2.06 \\
16.86\end{array}$ & $\begin{array}{c}64.03 \% \\
-1.69 \\
17.30\end{array}$ & $\begin{array}{c}28.78 \% \\
6.07 \\
18.34\end{array}$ \\
\hline $\mathrm{LC}$ & $\begin{array}{c}\% \text { stud. } \\
m \\
\sigma\end{array}$ & $\begin{array}{c}10.34 \% \\
-61.31 \\
15.37\end{array}$ & $\begin{array}{c}4.31 \% \\
-68.57 \\
15.65\end{array}$ & $\begin{array}{c}11.51 \% \\
-62.95 \\
16.74\end{array}$ & $\begin{array}{l}2.16 \% \\
-45.24 \\
4.12\end{array}$ \\
\hline
\end{tabular}




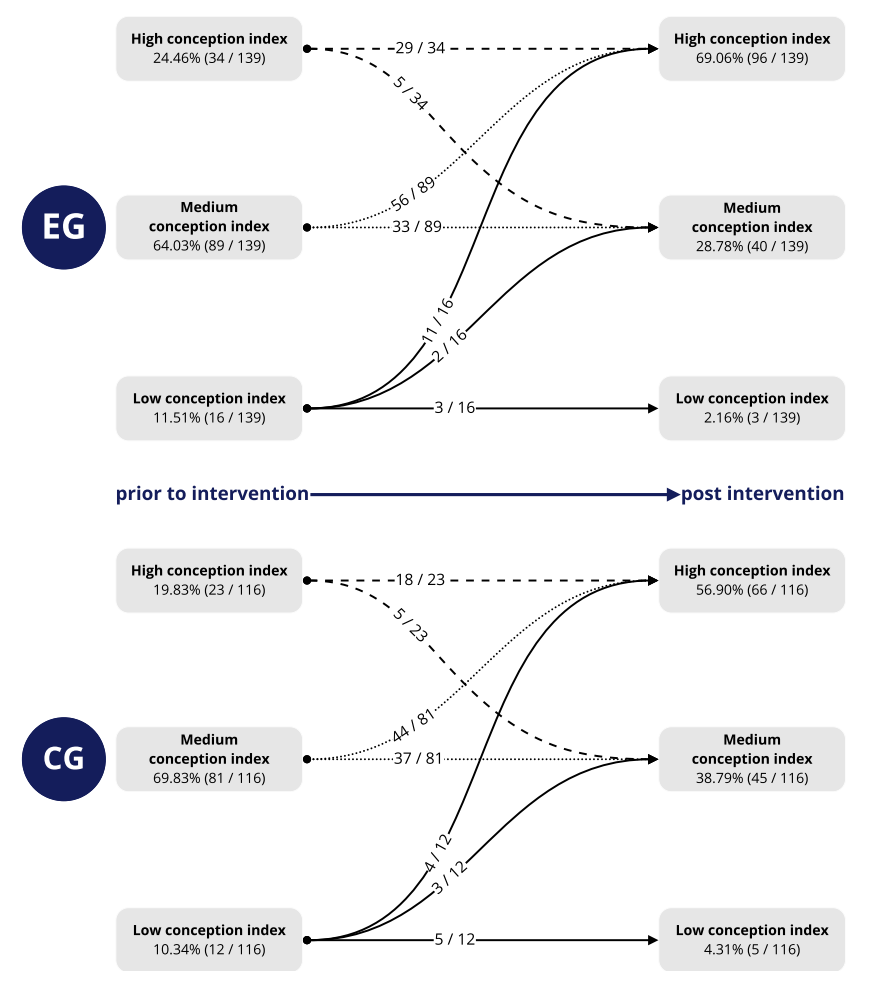

FIG. 11. Students' transitions between high HC, MC, and LC from pre- to post-test based on the students' results on the quantum physics' probability interpretation scale. It is shown how many students from each of the pretest groups $\mathrm{HC}, \mathrm{MC}$, and LC change to which group at the post-test point in time. For both survey time points, it is also indicates how many students are assigned to the three groups in total, both for EG and CG.

based on the post-test results. In the experimental group, however, this even applies to $63.80 \%$ of the students who were assigned to either the medium (56 out of 89) or the low conception index group (16 out of 139) at the pretest point in time.

\section{DISCUSSION}

With our quantum-optics based approach presented in this article we combine two central ideas: on the one hand, we want to give learners an insight into modern quantum physics experiments as they are used in today's research laboratories. On the other hand, we want to provide a learning environment that is conducive to the development of a conceptual understanding of the fundamental aspects of quantum physics at secondary school level (cf. Sec. II C.).

From research into students' difficulties, mixing concepts from classical and quantum physics has been found to be among the major obstacles for learners in developing conceptual understanding of quantum physics (cf. Sec. II B.). However, traditional quantum physics curricula at secondary schools today are still mostly guided by the historical development of quantum physics (cf. Sec. II A.), in which mechanistic and deterministic ideas are quite widespread.
Hence, in this study, we attempted to determine the effect of our teaching sequence based on coincidence and correlation experiments with heralded photons on learners' conceptions of quantum physics, compared to traditional introductory courses in quantum physics at German secondary schools.

The learners' conceptions of the probability interpretation of quantum physics and the quantum objects' properties and behavior were surveyed with the help of a conception questionnaire adapted from specialist literature in order to clarify the following research question: Are the students' conceptions on quantum objects' properties or behavior and quantum physics' probability interpretation less influenced by mechanistic or deterministic thinking when students are introduced to quantum physics via coincidence and correlation experiments with heralded photons, compared to the conceptions acquired by learners in a traditional introductory quantum physics course?

Mechanistic particle images of quantum objects, have been reported in various empirical studies on learners' conceptions of quantum physics [22,86-88]. Such conceptions are often accompanied by trajectory notions which are particularly noticeable in the context of atomic models [89,90]. Often learners assume that quantum objects move along well-defined trajectories unknown to the observer [91] having in mind mechanical paths. As a matter of fact, in our study, we find a statistically significant difference between the conception indices on quantum objects' properties and behavior of control $(m=32.71$, $\sigma=34.87)$ and experimental groups' students $(m=42.65$, $\sigma=38.36)$ from a $t$ test $[t(253)=-2.146, p=0.033$; $d=0.27$, leading to a rejection of the null hypothesis $H_{0}^{1}$ in the context of this study.

We believe that the origin of this observation can be found in the way that is talked about quantum physics throughout the course of our quantum-optics based teaching concept. Here, the reference discipline for quantum physics is optics rather than classical mechanics. Thus, discussing about mechanistic particles and their trajectories becomes superfluous. Against the backdrop of the PCA results (cf. Sec. V B), at least the learners' conceptions of quantum objects' properties indeed seem to be predominantly detached from mechanistic thinking (cf. Table VII). With regard to the behavior of quantum objects, on the other hand, mechanistic ways of thinking still seem to remain steadfastly in the learners' conceptions, despite participation in the quantum-optics based approach (cf. Table VII). In any case, introducing learners to quantum physics in the context of experiments with heralded photons seems to have advantages over the traditional curriculum based on quantum physics' historical development.

The rejection of deterministic notions is a significant hurdle for learners to overcome in quantum physics learning. Learners' understandings and conceptions about probability interpretation of quantum physics have been discussed in numerous papers and for different target groups [92-95]. The example of single photons at the beam splitter, as 
suggested in our quantum optics approach, shows quantum physics' statistical character clearly. While for the single photon, one cannot predict whether it will be detected at the detector at the reflected or the transmitted output, a prediction can be made for the observation of many such experimental realizations: An equal distribution between reflected and transmitted will occur. For this reason, we believe that the probability interpretation of quantum physics is conveyed in a quantum physics teaching approach that is oriented toward coincidence and correlation experiments with heralded photons in a particularly clearly manner. Our questionnaire investigation reveals that in both the traditional quantum physics instruction and the quantum optics-based concept, many students have gained an idea about the statistical character. For example, 52.6\% of the students in the control group agree with the statement

\section{I cannot make statements about the behavior of single photons inside the interferometer. I can only make statements about the statistical behavior of many sim- ilarly prepared photons (cf. Table III),}

while it is rejected by $19.0 \%$ of respondents in the control group. However, in the experimental group, this even applies to $66.2 \%$ of the learners (10.1\% rejection). Together with the other items on the probability interpretation of quantum physics (cf. Table III), our study provides evidence that quantum physics courses based on quantum optical experiments may be better suited to detach learners' conceptions from deterministic thinking: we find a statistically significant difference between the control group ( $m=34.98, \sigma=42.00)$ and experimental group $(m=50.51, \sigma=39.24)$ from a $t$ test $[t(253)=-3.049$, $p=0.003 ; d=0.38$ ], leading to a rejection of the null hypothesis $H_{0}^{2}$ in the context of this study.

In summary, we believe that the origin of this observation can be found in the detailed treatment of the experiment with single photons at the optical beam splitter during our quantum-optics based teaching sequence: using one of the interactive screen experiments, students realize that photons are always measured at exactly one output of the beam splitter. In this way, they learn very directly about fundamental concepts of quantum physics, such as the superposition of states or the measurement process. We consider the superposition of quantum mechanical states to be the core concept that students need to understand in order to discard deterministic ways of thinking. Introducing learners to quantum physics in the context of experiments with heralded photons seems particularly suitable for this purpose, against the backdrop of the results reported here.

\section{LIMITATIONS AND CONCLUSION}

In this paper, we reported the results of a comparative study of learning quantum physics in secondary schools. A traditional German quantum physics course (control group, five classes, 116 students) was compared with a modern teaching concept based on coincidence and correlation experiments with heralded photons (experimental group, six classes, 139 students), providing first empirical insights into the effects that quantum optics teaching proposals may have for secondary school teaching. Using a questionnaire on students' conceptions adapted from specialist literature, we find statistically significantly higher conception indices for the experimental group learners with regards to the quantum objects' properties and behavior $(d=0.27)$ and quantum physics' probability interpretation $(d=0.38)$.

In the future, further investigations have to follow, on the one hand, with respect to the verification and validation of the results reported here, and on the other hand with respect to the empirical investigation of learning processes of secondary school students in quantum optics-based introductory quantum physics courses more generally.

In order to tackle limitations of the study presented in this article, we need to concretely identify the learners' conceptions of the various quantum physics aspects (e.g., photons, property position, probability interpretation) developed in the context of quantum optics-based introductory courses beyond conception indices alone. We think these conceptions should be investigated using qualitative methods (e.g. interviews, group discussions), and need to be compared to those resulting from traditional introductory quantum physics courses in schools as they are reported from specialist literature [74]. In addition, it is necessary to clarify to what extent learners' conceptions are context dependent after an introductory course into quantum physics, using coincidence and correlation experiments with heralded photons. For example, for determining whether quantum optics-based teaching approaches to quantum physics are indeed preferable to traditional approaches, it seems crucial to investigate how students' conceptions change when one changes the context, e.g., from single photons in the interferometer to single electrons at the double slit.

Concerning the empirical investigation of learning processes of secondary school students in quantum optics-based introductory quantum physics courses in general, the following remark is important for us: with the study presented here, we do not enable generalizing conclusions about the learning effectiveness of quantum optics-based teaching concepts in general and in comparison to traditional teaching sequences in particular. However, we find that our results can mark the starting point for further similar studies, for instance with larger samples and test instruments that can validly assess learners' conceptual understanding of quantum physics, e.g., Refs. [34,68-73]. The difficulty here is to use such test instruments with which a comparison of quantum optics-based teaching proposals and traditional introductory courses is possible at all, e.g., with respect to learning effectiveness.

For this, it seems to make sense to pick out the concepts of quantum physics which, according to the community, should be taught in quantum physics classes at secondary 
schools in any case [96]. Future work could then focus on comparing students' acquired understanding of these quantum physics concepts in different settings (traditional teaching approaches vs quantum optics-based approaches).

\section{ACKNOWLEDGMENTS}

We thank the teachers and their classes for participating in our study. This study was funded by the Emerging Talents Initiative (University of Erlangen, Germany).

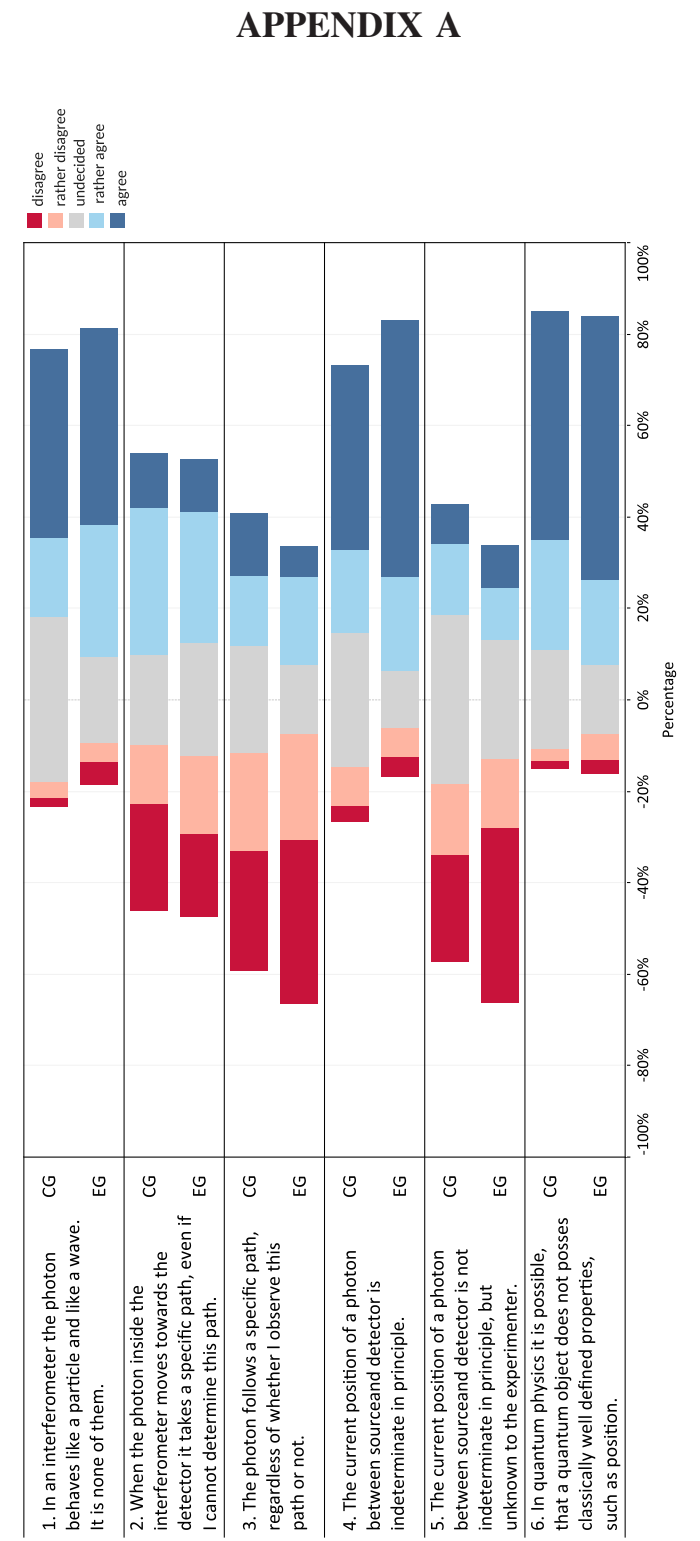

FIG. 12. Diverging stacked bar chart visualizing the distribution of student ratings on all items of the scale on the quantum objects' properties and behavior (cf. Table II).

\section{APPENDIX B}

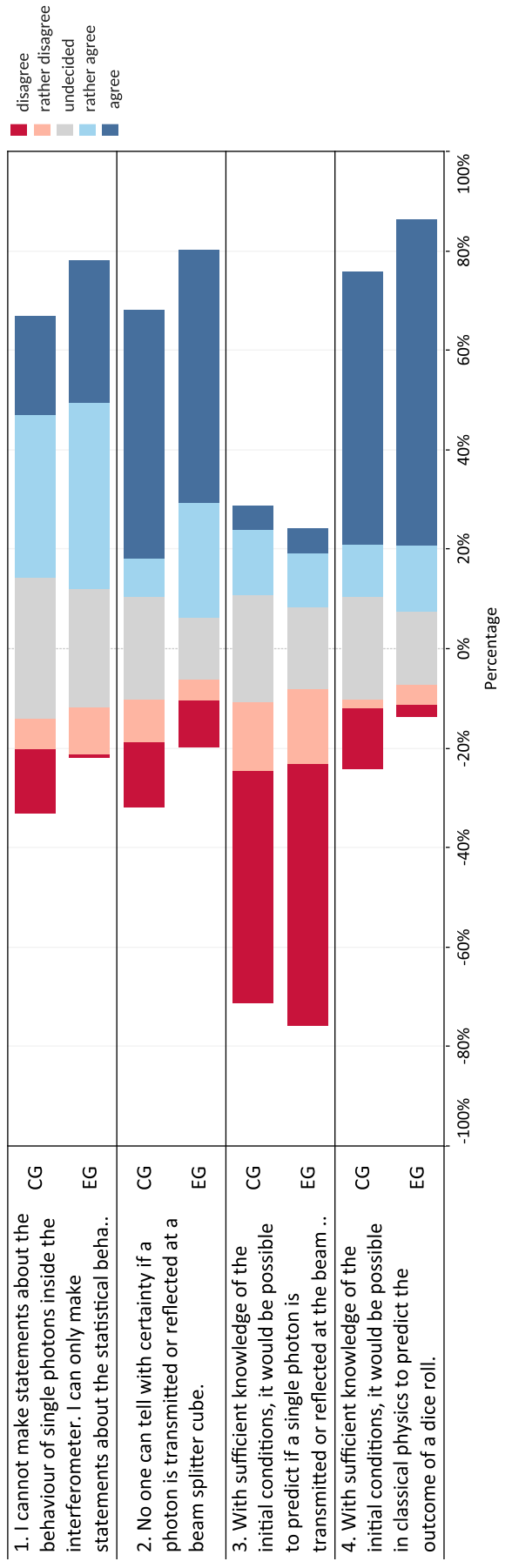

FIG. 13. Diverging stacked bar chart visualizing the distribution of student ratings on all items of the scale on the probability interpretation of quantum physics (cf. Table III). 
[1] P. Koczyk, P. Wiewior, and C. Radzewicz, Photon counting statistics-Undergraduate experiment, Am. J. Phys. 64, 240 (1996).

[2] A. C. Funk and M. Beck, Sub-Poissonian photocurrent statistics: Theory and undergraduate experiment, Am. J. Phys. 65, 492 (1997).

[3] C. H. Holbrow, E. J. Galvez, and M. Parks, Photon quantum mechanics and beam splitters, Am. J. Phys. 70, 260 (2002).

[4] E. J. Galvez, C. H. Holbrow, M. J. Pysher, J. W. Martin, N. Courtemanche, L. Heilig, and J. Spencer, Interference with correlated photons: Five quantum mechanics experiments for undergraduates, Am. J. Phys. 73, 127 (2005).

[5] D. Dehlinger and M. W. Mitchell, Entangled photons, nonlocality, and Bell inequalities in the undergraduate laboratory, Am. J. Phys. 70, 903 (2002).

[6] D. Dehlinger and M.W. Mitchell, Entangled photon apparatus for the undergraduate laboratory, Am. J. Phys. 70, 898 (2002).

[7] J. J. Thorn, M. S. Neel, V. W. Donato, G. S. Bergreen, R.E. Davies, and M. Beck, Observing the quantum behavior of light in an undergraduate laboratory, Am. J. Phys. 72, 1210 (2004).

[8] P. Bronner, A. Strunz, C. Silberhorn, and J.-P. Meyn, Demonstrating quantum random with single photons, Eur. J. Phys. 30, 1189 (2009).

[9] E. J. Galvez, Quantum optics laboratories for teaching quantum physics, in Proc. SPIE 11143, Fifteenth Conference on Education and Training in Optics and Photonics: ETOP 2019, 111431A, https://doi.org/10.1117/ 12.2523843.

[10] B. J. Pearson and D. P. Jackson, A hands-on introduction to single photons and quantum mechanics for undergraduates, Am. J. Phys. 78, 471 (2010).

[11] E. Dederick and M. Beck, Exploring entanglement with the help of quantum state measurement, Am. J. Phys. 82, 962 (2014).

[12] J. M. Ashby, P. D. Schwarz, and M. Schlosshauer, Delayed-choice quantum eraser for the undergraduate laboratory, Am. J. Phys. 84, 95 (2016).

[13] M. R. Coleman, K. G. Ingalls, J. T. Kavulich, S. J. Kemmerly, N. C. Salinas, E. V. Ramirez, and M. Schlosshauer, Assessing randomness with the aid of quantum state measurement, Am. J. Phys. 88, 238 (2020).

[14] H. J. Kimble, M. Dagenais, and L. Mandel, Photon Antibunching in Resonance Fluorescence, Phys. Rev. Lett. 39, 691 (1977)

[15] R. Hanbury Brown and R. Q. Twiss, Correlation between Photons in two Coherent Beams of Light, Nature (London) 177, 27 (1956).

[16] P. L. Kelley and W. H. Kleiner, Theory of Electromagnetic Field Measurement and Photoelectron Counting, Phys. Rev. 136, A316 (1964).

[17] L. Mandel and E. Wolf, Coherence properties of optical fields, Rev. Mod. Phys. 37, 231 (1965).

[18] P. Grangier, G. Roger, and A. Aspect, Experimental evidence for a photon anticorrelation effect on a beam splitter: A new light on single-photon interferences, Europhys. Lett. 1, 173 (1986).
[19] E. Marshman and C. Singh, Investigating and improving student understanding of quantum mechanics in the context of single photon interference, Phys. Rev. Phys. Educ. Res. 13, 010117 (2017).

[20] H. Fischler and M. Lichtfeldt, Modern physics and students' conceptions, Int. J. Sci. Educ. 14, 181 (1992).

[21] D. F. Styer, Common misconceptions regarding quantum mechanics, Am. J. Phys. 64, 31 (1996); 64, 1202 (1996).

[22] A. Mashhadi and B. Woolnough, Insights into students' understanding of quantum physics: visualizing quantum entities, Eur. J. Phys. 20, 511 (1999).

[23] G. Ireson, The quantum understanding of pre-university physics students, Phys. Educ. 35, 15 (2000).

[24] E. Marshman and C. Singh, Framework for understanding the patterns of student difficulties in quantum mechanics, Phys. Rev. ST Phys. Educ. Res. 11, 020119 (2015).

[25] C. Singh and E. Marshman, Review of student difficulties in upper-level quantum mechanics, Phys. Rev. ST Phys. Educ. Res. 11, 020117 (2015).

[26] G. Zhu and C. Singh, Improving students' understanding of quantum measurement. I. Investigation of difficulties, Phys. Rev. ST Phys. Educ. Res. 8, 010117 (2012).

[27] C. Singh, Student difficulties with quantum mechanics formalism, AIP Conf. Proc. 883, 185 (2007).

[28] G. Zhu and C. Singh, Surveying students' understanding of quantum mechanics in one spatial dimension, Am. J. Phys. 80, 252 (2012).

[29] P. Bitzenbauer and J.-P. Meyn, A new teaching concept on quantum physics in secondary schools, Phys. Educ. 55, 055031 (2020).

[30] P. Bitzenbauer and J.-P. Meyn, Fostering students' conceptions about the quantum world-results on an interview study, Prog. Sci. Educ. 4, 40 (2021).

[31] H. K. E. Stadermann, E. van den Berg, and M. J. Goedhart, Analysis of secondary school quantum physics curricula of 15 different countries: Different perspectives on a challenging topic, Phys. Rev. ST Phys. Educ. Res. 15, 010130 (2019).

[32] M. S. Ubben and S. Heusler, Gestalt and functionality as independent dimensions of mental models in science, Res. Sci. Educ. (2019).

[33] R. V. Olsen, Introducing quantum mechanics in the upper secondary school: a study in Norway, Int. J. Sci. Educ. 24, 565 (2002).

[34] S. B. McKagan, K. K. Perkins, and C.E. Wieman, Design and validation of the Quantum Mechanics Conceptual Survey, Phys. Rev. Phys. Educ. Res. 6, 020121 (2010).

[35] G. Kalkanis, P. Hadzidaki, and D. Stavrou, An instructional model for a radical conceptual change towards quantum mechanics concepts, Sci. Educ. 87, 257 (2003).

[36] C. Stefani and G. Tsaparlis, Students' levels of explanations, models, and misconceptions in basic quantum chemistry: A phenomenographic study, J. Res. Sci. Teach. 46, 520 (2009).

[37] J. Petri and H. Niedderer, A learning pathway in high school level quantum atomic physics, Int. J. Sci. Educ. 20, 1075 (1998). 
[38] K. S. Taber, Learning quanta: Barriers to stimulating transitions in student understanding of orbital ideas, Sci. Educ. 89, 94 (2005).

[39] K. Mannila, I. T. Koponen, and J. A. Niskanen, Building a picture of students' conceptions of wave- and particle-like properties of quantum entities, Eur. J. Phys. 23, 45 (2002).

[40] P. Bitzenbauer, Quantenoptik an Schulen-Studie im Mixed-Methods Design zur Evaluation des Erlanger Unterrichtskonzepts zur Quntenoptik (Logos Verlag, Berlin, 2020).

[41] A. Dutt, Making the transition from classical to quantum physics, Teach. Sci. 57, 33 (2011).

[42] D. C. Burnham and D. L. Weinberg, Observation of Simultaneity in Parametric Production of Optical Photon Pairs, Phys. Rev. Lett. 25, 84 (1970).

[43] P. G. Kwiat, E. Waks, A. G. White, I. Appelbaum, and P. H. Eberhard, Ultrabright source of polarizationentangled photons, Phys. Rev. A 60, R773(R) (1999).

[44] G. Brida, I. P. Degiovanni, M. Genovese, A. Migdall, F. Piacentini, S. V. Polyakov, and I. R. Berchera, Experimental realization of a low-noise heralded single-photon source, Opt. Exp. 19, 1484 (2011).

[45] M. D. Eisaman, J. Fan, A. Migdall, and S. V. Polyakov, Invited Review Article: Single-photon sources and detectors, Rev. Sci. Instrum. 82, 071101 (2011).

[46] D. Branning, S. Bhandari, and M. Beck, Low-cost coincidence-counting electronics for undergraduate quantum optics, Am. J. Phys. 77, 667 (2009).

[47] M. Beck and E. Galvez, Quantum optics in the undergraduate teaching laboratory, in Conference on Coherence and Quantum Optics, OSA Technical Digest (CD) (Optical Society of America, Washington, DC, 2007), paper CSuA4

[48] M. Facao, A. Lopes, A. L. Silva, and P. Silva, Computer simulation for calculating the second-order correlation function of classical and quantum light, Eur. J. Phys. 32, 925 (2011).

[49] J. Kirstein and V. Nordmeier, Multimedia representation of experiments in physics, Eur. J. Phys. 28, S115 (2007).

[50] J. Kirstein, S. Haase, T. Mühlenbruch, and V. Nordmeier, 20 Jahre Interaktive Bildschirmexperimente, PhyDidBBeiträge zur Frühjahrstagung 2016, http://www.phydid .de/index.php/phydid-b/article/view/739/872 (2016).

[51] P. Bronner, A. Strunz, C. Silberhorn, and J.-P. Meyn, Interactive screen experiments with single photons, Eur. J. Phys. 30, 345 (2009).

[52] C. K. Hong and L. Mandel, Theory of parametric frequency down conversion of light, Phys. Rev. A 31, 2409 (1985).

[53] A. Donhauser, P. Bitzenbauer, and J.-P. Meyn, Von Schneeund Elektronenlawinen: Entwicklung eines Erklärvideos zu Einzelphotonendetektoren, PhyDidBBeiträge zur Frühjahrstagung 2020, http://www.phydid .de/index.php/phydid-b/article/view/1021 (2020).

[54] R. Müller and H. Wiesner, Teaching quantum mechanics on an introductory level, Am. J. Phys. 70, 200 (2002).

[55] G. Passante, P. J. Emigh, and P. S. Shaffer, Student ability to distinguish between superposition states and mixed states in quantum mechanics, Phys. Rev. ST Phys. Educ. Res. 11, 020135 (2015).
[56] S. Prasad, M. O. Scully, and W. Martienssen, A quantum description of the beam-splitter, Opt. Commun. 62, 139 (1987).

[57] S. A. Ramakrishna, A. Bandyopadhyay, and J. Rai, A dissipative quantum mechanical beam-splitter, Opt. Express 2, 29 (1998).

[58] R. Müller and O.S. Mishina, milq-Quantum Physics in Secondary School, arXiv.org/abs/2012.15162v2.

[59] L. E. Ballentine, The statistical interpretation of quantum mechanics, Rev. Mod. Phys. 42, 358 (1970).

[60] R. Scholz, S. Wessnigk, and K.-A. Weber, A classical to quantum transition via key experiments, Eur. J. Phys. 41, 055304 (2020).

[61] D. G. C. Jones, Teaching modern physics-misconceptions of the photon that can damage understanding, Phys. Educ. 26, 93 (1991).

[62] A. Hobson, Electrons as field quanta: A better way to teach quantum physics in introductory general physics courses, Am. J. Phys. 73, 630 (2005).

[63] A. Hobson, Teaching quantum physics without paradoxes, Phys. Teach. 45, 96 (2007).

[64] V. Spatz, M. Hopf, T. Wilhelm, C. Waltner, and H. Wiesner, Introduction to Newtonian mechanics via twodimensional dynamics-The effects of a newly developed content structure on German middle school students, Eur. J. Sci. Math. Ed. 8, 71 (2020).

[65] J.-P. Burde and T. Wilhelm, Teaching electric circuits with a focus on potential differences, Phys. Rev. Phys. Educ. Res. 16, 020153 (2020).

[66] R. Müller, Quantenphysik in der Schule (Logos Verlag, Berlin, 2003).

[67] K. Heller and C. Perleth, Kognitiver Fähigkeitstest für 4. bis 12. Klassen, Revision: KFT 4-12+R (Betz Test, Göttingen, 2000).

[68] E. Cataloglu and R. W. Robinett, Testing the development of student conceptual and visualization understanding in quantum mechanics through the undergraduate career, Am. J. Phys. 70, 238 (2002).

[69] S. Wuttiprom, M. D. Sharma, I. Johnston, R. Chitaree, and C. Soankwan, Development and use of a conceptual survey in introductory quantum physics, Int. J. Sci. Educ. 31, 631 (2009).

[70] S. Goldhaber, S. J. Pollock, M. Dubson, P. Beale, and K. K. Perkins, Transforming upper-division quantum mechanics: Learning goals and assessment, in Proceedings of the 2009 Physics Education Research Conference, Ann Arbor, MI (AIP, New York, 2009), 145-148.

[71] H. Sadaghiani and S. J. Pollock, Quantum mechanics concept assessment: Development and validation study, Phys. Rev. ST Phys. Educ. Res. 11, 010110 (2015).

[72] S. di Uccio, A. Colantonio, S. Galano, I. Marzoli, F. Trani, and I. Testa, Design and validation of a two-tier questionnaire on basic aspects in quantum mechanics, Phys. Rev. Phys. Educ. Res. 15, 010137 (2019).

[73] E. Marshman and C. Singh, Validation and administration of a conceptual survey on the formalism and postulates of quantum mechanics, Phys. Rev. Phys. Educ. Res. 15, 020128 (2019).

[74] K. Krijtenburg-Lewerissa, H. J. Pol, A. Brinkman, and W. R. van Joolingen, Insights into teaching quan- 
tum mechanics in secondary and lower undergraduate education, Phys. Rev. Phys. Educ. Res. 13, 010109 (2017).

[75] A. Mashhadi and B. Woolnough, Cognitive mapping of advanced level physics students' conceptions of quantum physics, in Proceedings of the Conference on Educational Research (Australian Association for Research in Education), Singapore (1996), https://files.eric.ed.gov/fulltext/ ED414195.pdf.

[76] G. Ireson, A multivariate analysis of undergraduate physics students' conceptions of quantum phenomena, Eur. J. Phys. 20, 193 (1999).

[77] J. Cohen, Statistical Power Analysis for the Behavioral Sciences (Lawrence Erlbaum Associates, New York, 1988).

[78] N. Robbins and R. Heiberger, Plotting Likert and other rating scales, in Proceedings of the 2011 Joint Statistical Meeting (2011), pp. 1058, https://www.montana.edu/msse/ Data_analysis/Likert\%20Survey\%20Graphs.pdf.

[79] H. Kaiser, The application of electronic computers to factor analysis, Educ. Psychol. Meas. 20, 141 (1960).

[80] J. D. Brown, Choosing the right type of rotation in PCA and EFA, Shiken: JALT Testing and Evaluation SIG Newsletter 13, 20 (2009), https://hosted.jalt.org/test/PDF/ Brown31.pdf.

[81] L. J. Cronbach, Coefficient alpha and the internal structure of tests, Psychometrika, 16, 297 (1951).

[82] C. Fornell and D. F. Larcker, Evaluating structural equation models with unobservable variables and measurement error, J. Market. Res. 18, 39 (1981).

[83] J. F. Hemphill, Interpreting the magnitudes of correlation coefficients, Am. Psychol. 58, 78 (2003).

[84] R. Eisinga, M. te Grotenhuis, and B. Pelzer, The reliability of a two-item scale: Pearson, Cronbach, or SpearmanBrown?, Int. J. Public Healt., 58, 637 (2013).

[85] L. W. Lam, Impact of competitiveness on salespeople's commitment and performance, J. Business Res. 65, 1328 (2012).
[86] M. Bormann, Das Schülervorverständnis zu Elektronen, Vorträge der DPG-Gießen 1986, 227 (1986).

[87] H. Wiesner, Verständnisse von Leistungskursschülern über Quantenphysik, Physik in der Schule 34, 95 (1996).

[88] M. Ayene, J. Kriek, and B. Damtie, Wave-particle duality and uncertainty principle: Phenomenographic categories of description of tertiary physics students' depictions, Phys. Rev. Phys. ST Educ. Res. 7, 020113 (2011).

[89] M. Lichtfeldt, Schülervorstellungen in der Quantenphysik und ihre möglichen Veränderungen durch Unterricht, Reihe Naturwissenschaften und Unterricht, Vol. 15 (Essen, Westarp, 1992).

[90] T. Bethge, Aspekte des Schülervorverständnisses zu grundlegenden Begriffen der Atomphysik, Dissertation, Universität Bremen, 1988.

[91] M.S. Ubben, Typisierung des Verständnisses mentaler Modelle mittels empirischer Datenerhebung am Beispiel der Quantenphysik (Logos Verlag, Berlin, 2020).

[92] L. Bao and E. Redish, Understanding probabilistic interpretations of physical systems: A prerequisite to learning quantum physics, Am. J. Phys. 70, 210 (2002).

[93] D. Domert, C. Linder, and A. Ingerman, Probability as a conceptual hurdle to understanding one-dimensional quantum scattering and tunnelling, Eur. J. Phys. 26, 47 (2005).

[94] M. Wittmann, R. Steinberg, and E. Redish, Investigating student understanding of quantum physics: Spontaneous models of conductivity, Am. J. Phys. 70, 218 (2002).

[95] C. Singh, M. Belloni, and W. Christian, Improving students' understanding of quantum mechanics, Phys. Today 59, No. 843 (2006).

[96] K. Krijtenburg-Lewerissa, H. J. Pol, A. Brinkman, and W. R. van Joolingen, Key topics for quantum mechanics at secondary schools: A Delphi study into expert opinions, Int. J. Sci. Educ. 41, 349 (2019). 